\title{
Subsurface Failure in Spherical Bodies: A Formation Scenario for Linear Troughs on
} Vesta's Surface

\author{
A.M. Stickle ${ }^{1,2 *}$, P.H. Schultz ${ }^{1}$, D.A. Crawford ${ }^{3}$ \\ ${ }^{1}$ Department of Geological Sciences, Brown University, Providence, RI 02912 \\ ${ }^{2}$ Present address: Johns Hopkins University Applied Physics Laboratory, Laurel, MD 20723 \\ ${ }^{3}$ Sandia National Laboratories, Albuquerque, NM 87185 \\ *corresponding author: Angela M. Stickle \\ Johns Hopkins University Applied Physics Laboratory \\ 11101 Johns Hopkins Rd. \\ Mail Stop: 200-W230 \\ Laurel, MD 20723 \\ Email: angela_stickle@alumni.brown.edu \\ Phone: (240) 228.3822
}

Keywords: Asteroid Vesta; Impact processes; Cratering 


\begin{abstract}
Many asteroids in the solar system exhibit unusual, linear features on their surface. The Dawn mission recently observed two sets of linear features on the surface of the asteroid 4 Vesta. Geologic observations indicate that these features are related to the two large impact basins at the south pole of Vesta, though no specific mechanism of origin has been determined. Further, the orientation of the features is offset from the center of the basins. Experimental and numerical results reveal that the offset angle is a natural consequence of oblique impacts into a spherical target. Here we demonstrate that a set of shear planes develops in the subsurface of the body opposite to the point of first contact. These subsurface failure zones then propagate to the surface under combined tensile-shear stress fields after the impact to create sets of approximately linear faults on the surface. Comparison between the orientation of damage structures in the laboratory and failure regions within Vesta can be used to constrain impact parameters (e.g., the approximate impact point and likely impact trajectory).
\end{abstract}

\title{
1. Introduction
}

Comets and asteroids represent survivors of original planetary building blocks that record conditions of the early solar system. Nevertheless, nearly all asteroids observed close-up today have impact craters with diameters comparable to the body itself [e.g., Bottke et al., 2002]. The size of these craters implies that the interior of these asteroids must be highly fractured and damaged. Consequently, most small bodies underwent collisional, dynamical and thermal processes over the last $4.5 \mathrm{Ga}$.

Previous experimental and numerical work examined failure of spherical targets to better understand asteroid evolution, damage structures seen on asteroids, and limits for catastrophic disruption. Impact-induced failure within a spherical target contrasts with failure in a planar target due to curvature of the surface that focuses shock waves towards the center with incipient (if not total) failure at the farside [e.g., Rinehart 1960; Gault and Wedekind, 1969]. This phenomenon is important even for planetary bodies such as the Moon and Mercury [Schultz and Gault, 1975; Hughes et al., 1977; Schultz and Crawford, 2011], and Saturnian satellites [Bruesch and Asphaug, 2004; Moore et al., 2004].

Laboratory impacts into spherical targets have been used to better understand the effects of material properties on collisional outcomes of asteroids [e.g., Fujiwara et al., 1989; Nakamura and Fujiwara, 1991; Martelli et al., 1994; Holsapple et al., 2002]. Impact experiments indicate that impact angle, in conjunction with material properties and impact velocity, significantly affects collisional outcomes [Gault, 1973; Gault and Wedekind, 1978; Fujiwara and Tsukamoto, 1980]. Previous experimental studies show that catastrophic disruption of basalt and glass targets resulted in an intact fragment at the center of the original target (called the "core") [Gault and Wedekind, 1969; Fujiwara, 1986; Nakamura and Fujiwara, 1991]. This phenomenon is also observed in PMMA targets when they are struck at high velocity.

Laboratory experiments, however, are orders of magnitude smaller than collisions throughout the solar system. Thus, numerical modeling or scaling relationships [e.g., Housen et al., 1983; Holsapple, 1993; Davis et al., 1994] become increasingly important for understanding collisional histories of asteroids and larger terrestrial bodies. Hydrocode simulations of asteroid disruption were originally benchmarked by comparison with disruption of laboratory-scale spherical targets [e.g., Melosh et al., 1992; Benz and Asphaug, 1994; Asphaug, 1997; Melosh and Ryan, 1997; Ryan and Melosh, 1998; Benz and Asphaug, 1999]. Two-dimensional simulations were generally successful in reproducing fragment size distribution and mean ejecta 
speeds from laboratory experiments into basalt [Takagi et al., 1984; Hartmann, 1980; Nakamura and Fujiwara, 1991] and weak and strong mortar targets [Davis and Ryan, 1990].

Hydrocode simulations also revealed subsurface damage caused by impacts on asteroids [e.g., Asphaug, 1997; Nolan et al., 2001; Buczkowski et al., 2012; Bowling et al., 2013a,b; Ivanov and Melosh, 2013]. Asphaug et al. [1996] used the hydrocode SALE2D [Amsden et al., 1980] to examine subsurface damage on the asteroid Ida, and Asphaug [1997] provided similar analysis for the asteroid 4 Vesta. Their results indicated that stresses from large events into curved, finite target surfaces focus in regions far from the impact point, causing fracture throughout the body; this can be exacerbated by a non-spherical target [Asphaug et al. 1996]. Further, Asphaug et al. [1996] showed that the impactor responsible for the Vienna Regio region of Ida may have caused fracturing in Pola Regio. Spacecraft images of Pola Regio reveal sets of parallel to sub-parallel troughs and linear features in this region. The authors concluded that these linear features may be the result of the Vienna Regio impact, and that later impacts may have created stress waves that reactivated these ancient flaws such that the grooves appear fresh on the surface of Ida.

Over the last two decades, missions to asteroids and comets have become increasingly common [e.g., NEAR, Galileo, Deep Impact, Stardust, Hayabusa, EPOXI, Stardust-NExT, Dawn, Rosetta, OSIRIS-REx]. These missions reveal a great deal about the nature of small bodies in the solar system, including surface properties, morphologies, and shapes. Because internal structure and damage can be manifested on the surface of bodies [e.g., Asphaug et al., 1996; Buczkowski et al., 2008; Schultz and Crawford, 2011], an understanding of subsurface damage caused by impact is important for fully understanding data returned by these missions.

The present study first describes a new time-resolved experimental approach for assessing damage in spherical targets. This description is followed by a detailed comparison between subsurface damage in laboratory experiments and small-scale CTH simulations. We then discuss the results of CTH simulations on the scale of a particular asteroid: 4 Vesta. We finish with discussion of the damage structures in spherical targets, including formation mechanisms, differences with planar targets, and implications for asteroid evolution and future study.

\section{Methods and Approach}

Previous studies focused on planar polymethylmethacrylate (PMMA) targets in order to track the evolution of subsurface damage following oblique impacts for both impacts directly into PMMA [Stickle and Schultz, 2011; Stickle and Schulz, 2014] and impacts into layered PMMA and geologic targets [Stickle and Schultz, 2012; Stickle and Schultz, 2013]. At planetary scales, however, target curvature creates shock-wave interactions that significantly change the damage pattern (and process). Here, impacts into transparent PMMA spheres are directly compared with three-dimensional CTH models. These results, and the intuition gained from comparing small-scale models to experiments, then provide insights into extrapolations to large-scale planetary models.

\subsection{Laboratory Experiments}

A suite of laboratory experiments was performed at the Ames Vertical Gun Range (AVGR) at the NASA Ames Research Center in Mountain View, California. The AVGR is a two-stage light gas gun that achieves impact velocities from $\sim 0.5-7 \mathrm{~km} / \mathrm{s}$ (depending on projectile size). The barrel can rotate from $0-90^{\circ}$, in $15^{\circ}$ increments, allowing the target to stay oriented correctly with respect to gravity, which is especially important when studying impacts into 
granular or fluid targets. The targets are set up inside a $2.5-\mathrm{m}$ diameter vacuum chamber, so a wide variety of shapes and sizes can be accommodated. The vacuum chamber can be maintained at a vacuum level below 0.3 torr, or filled with different gasses to simulate various environments. High-speed cameras (up to 1 million frames per second) are used to record the impact events.

For this study, transparent PMMA spheres were used as the target. PMMA is a transparent acrylic that becomes opaque under high strain and allows tracking the evolution of impact-induced damage. At room temperature and high-strain rates, PMMA is brittle and has mechanical properties similar to that of the upper crust of the Earth, which makes it an ideal rock analog in high-rate laboratory experiments [de Joussineau et al., 2003; Rosakis et al., 1999, 2004, 2008; Rittel and Brill, 2008; Nasraoui et al., 2012]. PMMA, along with some other transparent polymeric materials, also exhibits birefringence, making it useful for studies of dynamic failure processes because stress fields can be mapped using photoelasticity techniques [e.g., de Joussineau et al., 2003; Rosakis et al., 1999; Rosakis, 2002; Misra et al., 2009]. While not a perfect match to a differentiated, rocky body, the brittle fracture processes that occur within these targets are the same that would be expected in brittle rock targets. Thus, this choice of target material allows the best of both worlds: brittle damage processes, and the ability to track those processes and the resulting damage evolution with time.

For this study, spherical targets with a $10-\mathrm{cm}$ diameter were placed on top of a thin $(0.3$ $\mathrm{cm})$ plastic cylinder protruding from a sand surface. Pyrex spheres $(0.635-\mathrm{cm}$ in diameter) impacted the target at $\sim 5.5 \mathrm{~km} / \mathrm{s}$ with impact angles ranging from $40^{\circ}-65^{\circ}$. The downward direction of impact minimized interactions with the thin cylinder supporting the sphere (evident in imaging and in lack of surface damage to the sphere). The evolution of the damage was tracked using two high-speed cameras (Shimadzu, HV-1) using frame rates of 250,000 to 500,000 frames per second over the first $200 \mu \mathrm{sec}$ after impact, sufficient time for the damage zones to finish growing. The frame rate of 500,000 frames per second also provided adequate time resolution to track the damage zone growth rate.

\subsection{CTH Models}

CTH is a multi-dimensional, multi-material, two-step Eulerian hydrocode model developed by Sandia National Laboratories in Albuquerque, New Mexico [McGlaun et al., 1990]. This code is used widely to model hypervelocity impacts into planetary surfaces because it is designed to model strong shock processes. The code also encompasses a variety of material models and equations of state (EOS).

All calculations for this study were performed in a three-dimensional rectangular coordinate system, which is necessary to model oblique impacts. The CTH models here are used to identify conditions of failure observed within the impacted spheres for identical impact parameters as the experiments. Adaptive Mesh Refinement (AMR) [Crawford, 1999; Crawford et al., 2006] is used to refine the mesh around high-pressure regions (i.e., the shock wave), while leaving other portions of the target at a lower resolution, which significantly reduces computational time. The maximum resolution for the laboratory-scale models is $0.026 \mathrm{~cm}(\sim 16$ cells per projectile radius (cppr)).

Sophisticated constitutive models are necessary for accurate models of material behavior undergoing differential stress. In these models, the Pyrex projectile is assumed to behave as a geologic material with a pressure-dependent yield surface. In order to track subsurface failure in PMMA, however, a slightly more complicated failure model is necessary. A Von Mises plasticity model is used to track the deformation, which is coupled with the Johnson-Cook 
Fracture (JCF) model. This model reveals regions of plastic failure (which are taken to be regions of shear deformation) within the PMMA sphere. The JCF model is a scalar damage model used to predict failure of materials [Johnson and Cook, 1985]. A damage parameter, D, ranges from 0 (pristine, undamaged material) to 1 (failed material), where $\mathrm{D}$ is a function of equivalent plastic strain rate, local value of plastic strain to fracture, pressure, local yield stress, temperature and loading path. In order to better understand the failure modes and to minimize the parameter space, $\mathrm{D}$ is assumed to be a function of the local value of plastic strain to fracture only. Shear failure (defined at $\mathrm{D}=1$ ) will occur when the material stresses exceed the yield stress and then the material undergoes a user-specified value of plastic strain. Here, we assume that the material has a yield stress of $120 \mathrm{MPa}$ and that failure occurs at $10 \%$ strain, after a previous study by Stickle and Schultz [2011].

The JCF model only tracks failure due to shear deformation. Nevertheless, this model is often used in conjunction with a separate spallation failure criterion in order to predict failure of pre-damaged material or material experiencing excess hydrostatic tension. For this study, however, shear and tensile failure are considered separately and a separate simulation is run to examine each process. Damage by extensional failure (spallation) occurs when the material undergoes stresses higher than the maximum tensile stress. In reality, later occurring shear can capitalize on zones under extension; hence, it would be useful to have an integrated model. Our approach, however, is to isolate and identify these two modes.

An equation of state relating pressure and volume is necessary in order to close the equation set. Here, a Mie-Grüneisen EOS is used for both Pyrex [Marsh, 1980] and PMMA (with values available within the CTH package libraries). The Mie-Grüneisen EOS provides a good representation of PMMA below $25 \mathrm{GPa}$, but can be used with confidence up to pressures of 100 $\mathrm{GPa}$. Because we are not examining the impact point, but rather areas far away that are at lower pressures, this EOS is a valid representation for our model. Table 1 summarizes the model parameters used in the CTH simulations.

Table 1. EOS and strength model parameters used in the CTH calculations

\begin{tabular}{|c|c|c|c|c|c|}
\hline Parameter & Pyrex $^{1}$ & PMMA $^{2}$ & Basalt & Dunite & Iron \\
\hline Density (g/cc) & 2.23 & 1.186 & 2.86 & 3.32 & 7.85 \\
\hline Sound speed $(\mathrm{km} / \mathrm{s})$ & 2 & 2.3 & 5.17 & 6.59 & 4.99 \\
\hline $\begin{array}{c}\text { Linear coefficient of the Us-up } \\
\text { Hugoniot curve: } \mathrm{S} 1\end{array}$ & 1.5 & 1.75 & -- & -- & -- \\
\hline $\begin{array}{l}\text { Quadratic coefficient of the Us- } \\
\text { up } \\
\text { Hugoniot curve: S2 }\end{array}$ & 0 & -0.13 & -- & -- & -- \\
\hline Gruneisen Parameter: $\Gamma$ & 1 & 0.91 & -- & -- & -- \\
\hline $\mathrm{Cv}(\mathrm{erg} / \mathrm{g} / \mathrm{eV})$ & $1 \times 10^{11}$ & $3.5 \times 10^{11}$ & -- & $1.35 \times 10^{11}$ & $5.16 \times 10^{10}$ \\
\hline Yield Strength (MPa) & 1000 & 120 & 1000 & 1000 & 1000 \\
\hline Yield Strength at $\mathrm{P}=0(\mathrm{MPa})$ & 2 & -- & 2 & 2 & \\
\hline Poisson's ratio & 0.26 & 0.37 & 0.26 & 0.26 & 0.28 \\
\hline Strain to Failure & -- & $10 \%$ & -- & -- & -- \\
\hline $\begin{array}{l}\text { Slope of the yield surface at } \\
\qquad P=0: d y / d p\end{array}$ & 0.5 & -- & 0.5 & 0.5 & -- \\
\hline
\end{tabular}




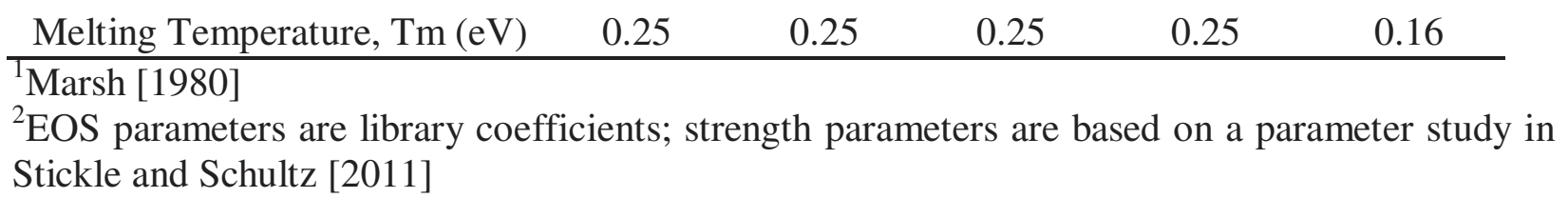

Laboratory-scale experiments provide important insights into processes occurring following oblique impacts and direct comparisons with numerical models establish confidence for larger scale applications. By comparing small-scale models with "ground truth" from experiments, the processes that are well-matched are easily identified. This comparison, then, provides confidence in understanding the processes revealed by planetary-scale simulations. Following the smallscale comparisons, the next step considered a $5 \mathrm{~km} / \mathrm{s}$ (the mean asteroidal impact velocity) impact into the differentiated asteroid 4 Vesta. This asteroid was modeled as a spherical, differentiated body with a diameter of $578 \mathrm{~km}$, and core size and thickness were assumed to follow the model of Ruzicka et al. [1997]. The diameter of the sphere was taken to be the longest axis of Vesta [Thomas et al., 1997; Ruzicka et al., 1997]. It is likely that Vesta did not originate as a spherical body, and that it was an oblate spheroid even prior to the large impacts at the south pole. However, because the original shape of Vesta is unknown, a sphere represents a good first step to understand the effect of the giant impacts and it is more readily compared to the small-scale experiments and simulations. The metallic core in these simulations has a diameter of $164 \mathrm{~km}$, and was modeled using the ANEOS equation of state (i.e., Analytic Equations of State) [Thompson and Lauson, 1984; Thompson, 1990] for iron and a Von Mises yield surface. The dunite mantle and basaltic crust have thicknesses of $165 \mathrm{~km}$ and $42 \mathrm{~km}$, respectively. The calculations used ANEOS for dunite, and a Sesame table for basalt. The selection of dunite and basalt provide a reasonable match for the composition of 4 Vesta [McCord et al., 1970; Binzel et al., 1997; Thomas et al., 1997]. The impactor was assumed to be an undifferentiated dunite sphere with a radius of $50 \mathrm{~km}$. Dunite and basalt are modeled as geologic materials with a pressure-dependent yield surface. Simulations varying the impactor and core size (for a smaller core and thicker crust/mantle based on new estimates from the Dawn mission [Russel et al., 2012]) did not significantly alter the results.

Davis et al. [1985] show that gravitational self-compression of material is important in understanding cratering processes on asteroids. Thus, the calculation also included self-gravity for the asteroid and the impactor based on a parallel implementation of the Barnes and Hut [1986] algorithm, as well as AMR. Recent models and analysis of data from the Dawn mission indicate that the surface gravity on Vesta ranges from $0.23-0.27 \mathrm{~m} / \mathrm{s}^{2}$ [Konopliv, et al. 2013; Bills et al. 2014; Ermakov et al. 2014]. Here, the asteroid was initialized at a constant temperature of $297 \mathrm{~K}$, with surface gravity $\sim 0.26 \mathrm{~m} / \mathrm{s}^{2}$ [Ruzicka et al. 1997]. Initial density, pressure and gravity profiles through the body are shown in Figure 1. The maximum resolution for the large-scale simulations was $2.5 \mathrm{~km}$ (20 cells across the projectile), which was adequate to capture shock compression, release and failure within the body. 

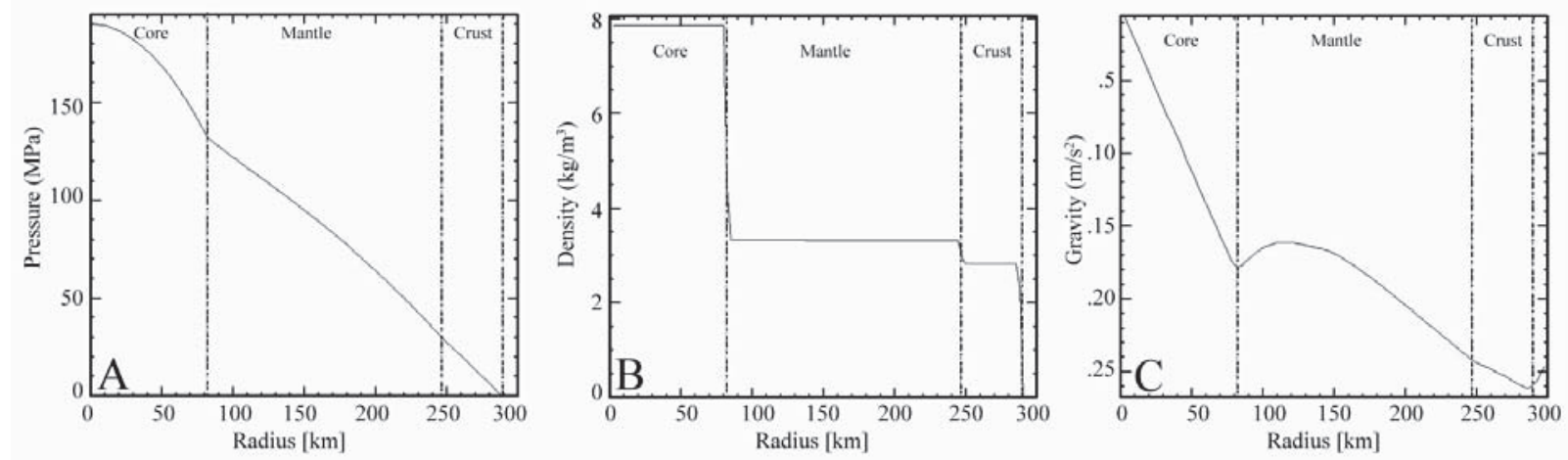

Figure 1. A) Pressure, B) density and C) gravity as a function of radius at time $=0$ for simulated asteroid 4 Vesta. Vertical dashed lines indicate transitions between crust, mantle and core. The asteroid was modeled as a $578-\mathrm{km}$ diameter differentiated body with a 164-km iron core, $165-\mathrm{km}$ thick dunite mantle and 42-km thick basaltic crust [after Ruzicka et al., 1997].

\section{Damage in a Spherical Target}

The laboratory experiments provide a clear view of damage growth as well as insight about particular damage structures, but it is sometimes difficult to understand all of the processes that are occurring following impact. Numerical models, however, track the state of the material following impact and the modes of deformation and damage growth and fill this gap in understanding. Conversely, materials deform in complicated ways, and material properties are not always well constrained. Instead, experiments provide an important reality check for the complicated numerical models and a way to benchmark our simulations. This section will first detail the results of laboratory experiments into spherical targets before detailing results of CTH models for comparison. The last portion of this section examines results of asteroid-scale impact simulations and how we can extrapolate and scale small-scale results to the giant Vestan impacts.

\subsection{Laboratory Experiment Results}

A suite of impact experiments was performed at the NASA Ames Vertical Gun Range examining the effect of impact angle on the initiation and growth of subsurface failure planes. Impact velocities were kept approximately constant $(5.5 \mathrm{~km} / \mathrm{s})$ and impact angle was varied between $40^{\circ}-65^{\circ}$.

A time sequence of typical failure evolution is shown in Figure 2. Directly following impact, damage begins to grow asymmetrically beneath the impact point, concentrated downrange. Approximately $24 \mu \mathrm{sec}$ after impact, a shallow subsurface haze forms downrange (about $90^{\circ}$ ) from the impact and is composed of small failure discs just below the surface. This hazy failure grows around the sphere, towards the impact point antipode. Around $40 \mu \mathrm{sec}$ after impact, a stalk of damage grows into the sphere from the farside of the target. This stalk grows up towards the center of the sphere, with the tip emerging around $60 \mu \mathrm{sec}$ as it grows above the haze boundary. Following this growth, planes of failure aligned perpendicular to the central column begin to grow. These planes are seen clearly in Figure 2 at $88 \mu \mathrm{sec}$ and continue to grow outward until about $100 \mu \mathrm{sec}$ after impact. Following damage formation, the finished structure resembles a blooming flower, and is termed a "tiered rosette" throughout the rest of the paper (this structure is also observed in Schultz and Crawford [2011], where the authors call it a "treelike" feature). Figure 3 provides a closer view of this structure (same experiment in Figure 2). Planes perpendicular to the central damage column, which points up towards the center of the 
sphere, comprise the "petals" of the tiered rosette structure. The orientation of these final damage structures depends somewhat on impact angle and velocity (Figure 4), but they all evolve in the same manner. Beneath the impact point, a large failure region grows. This region is centered on a point downrange from the impact point, common to most oblique impacts. Late in the damage growth process (following the completion of the subsurface damage growth), large pieces of the target spall from the impact-side of the target due to the small size of the target.

Figure 4 examines final damage structures, and how the orientation and location of the damage is dependent on impact angle. The bottom inset shows close-up views of the rosette structure, with the impact antipode marked for reference. For increasingly oblique impact angles, the damage planes are elongate in the downrange direction (also shown in Figure 2). The impact antipode is also marked in the inset for reference, and the impact point is shown in the upper inset. Note that the central damage column is centered around the impact point antipode, not the crater center, for oblique impacts. 

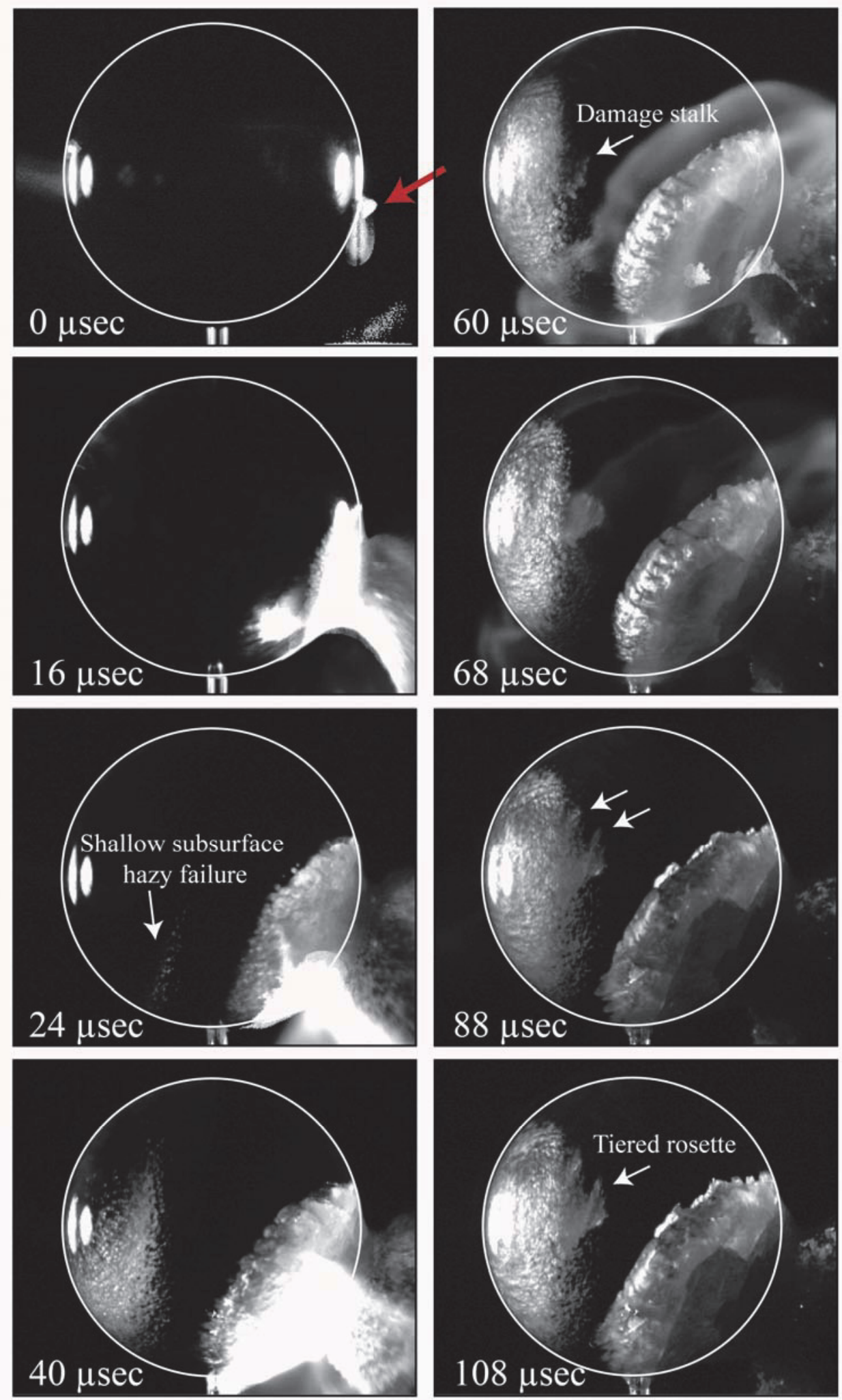

Figure 2. Time sequence showing the damage evolution within a spherical target. The projectile was a $0.635-\mathrm{cm}$ Pyrex sphere into a 10-cm PMMA sphere at an angle of 47 degrees and a velocity of $5.6 \mathrm{~km} / \mathrm{s}$. 


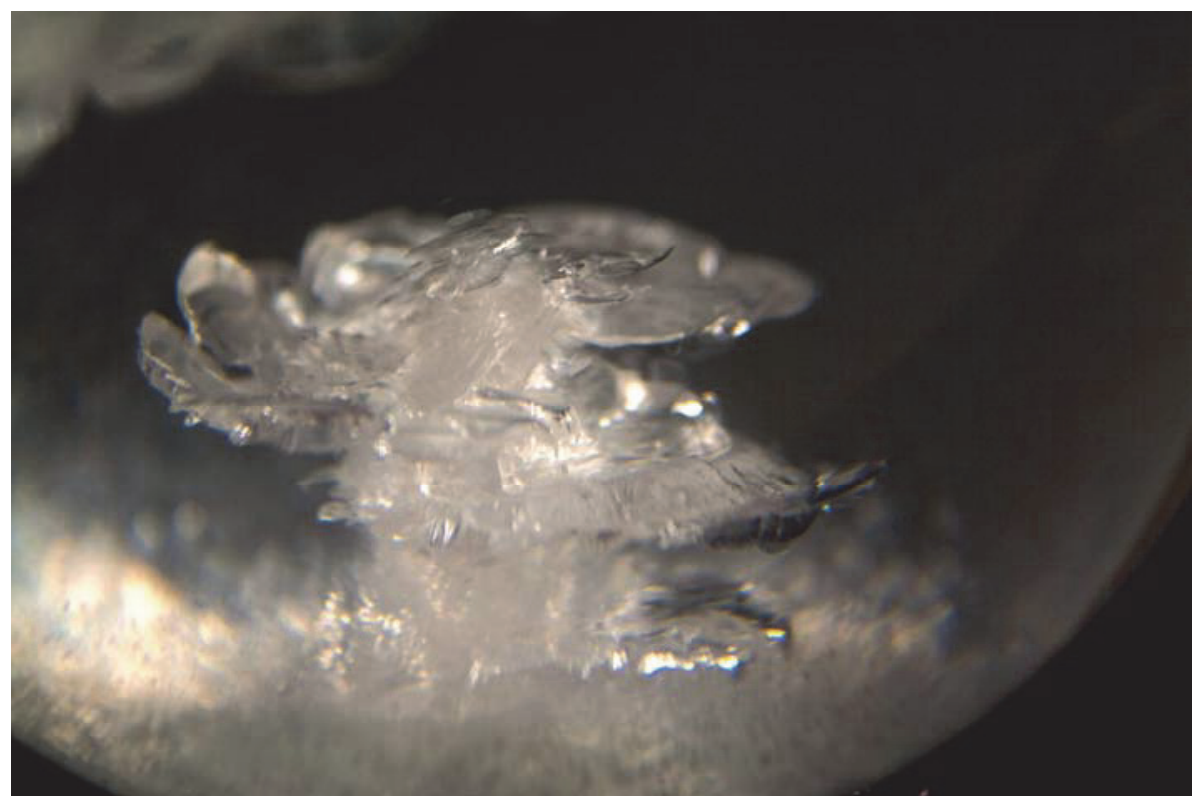

Figure 3. Close view of the "rosette" structure (planes extending from a central failure column) for the experiment shown in Figure 2. The "petals" form incomplete planes around the central damage column at various depths. 

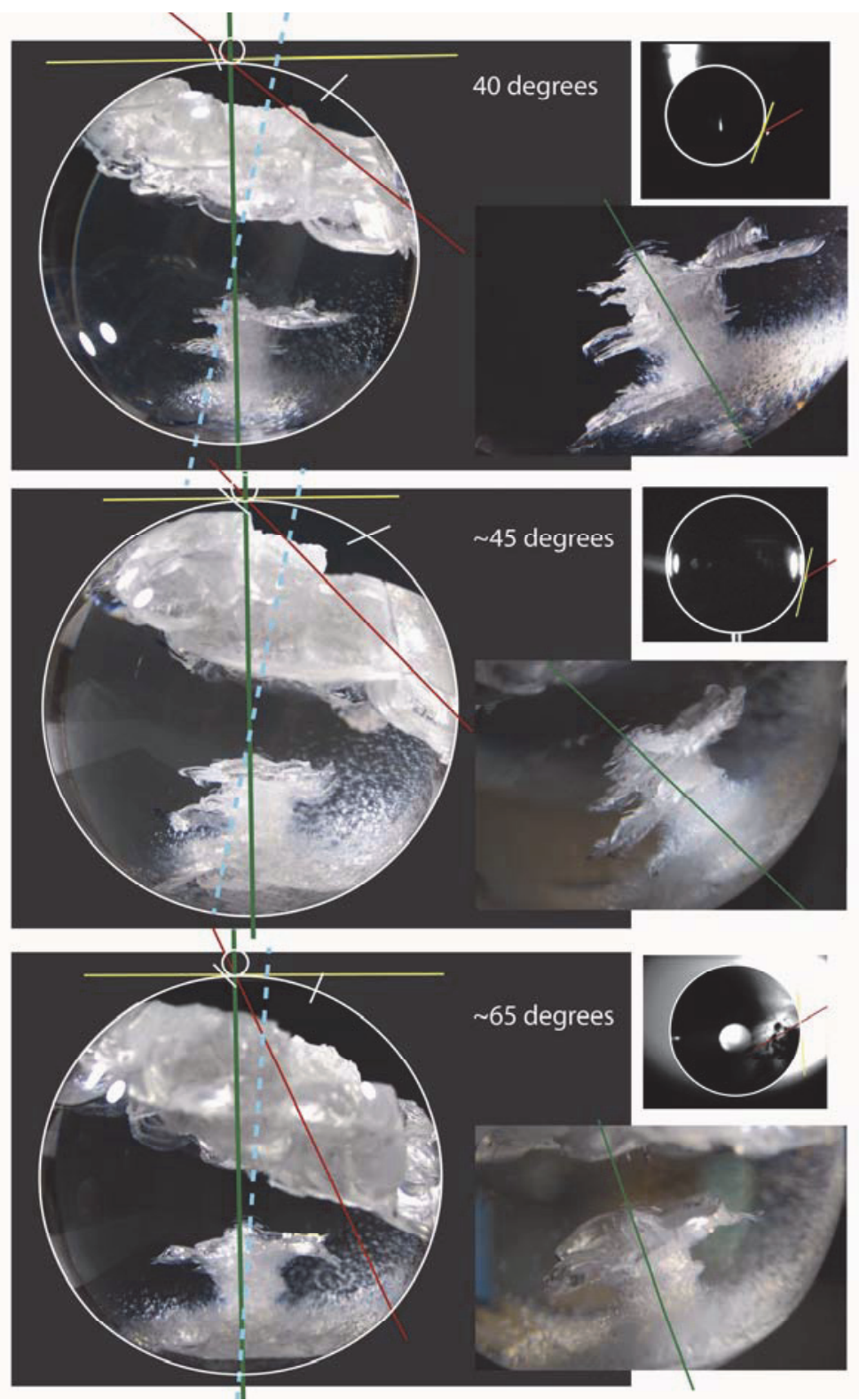

Figure 4. Comparison of final damage structures for three different impact angles. The red line shows the trajectory of the projectile, the light blue line shows the antipode to the crater center, and the green line indicates the antipode to the impact point. The approximate crater size, measured from high-speed images of the impact, is shown by the white hash marks. Top: impact of a $0.635-\mathrm{cm}$ Pyrex sphere into a 10-cm PMMA sphere at $5.4 \mathrm{~km} / \mathrm{s}$ at an impact angle of 40 degrees to the surface tangent plane. Middle: impact of a $0.635-\mathrm{cm}$ Pyrex sphere into a $10-\mathrm{cm}$ PMMA sphere at $5.6 \mathrm{~km} / \mathrm{s}$ at an impact angle of 47 degrees; (bottom) impact of a $0.635-\mathrm{cm}$ Pyrex sphere into a 10-cm PMMA sphere at $5.4 \mathrm{~km} / \mathrm{s}$ at 65 degrees. The insets show close-up views of the rosette structure. For increasingly oblique impact angles, the damage planes are elongate in the downrange direction (also shown in Figure 2). The impact antipode is also marked in the inset for reference, along with the impact point indicating impact angle and location. Note that the central damage column is centered around the impact point antipode, not the crater center, for oblique impacts. 


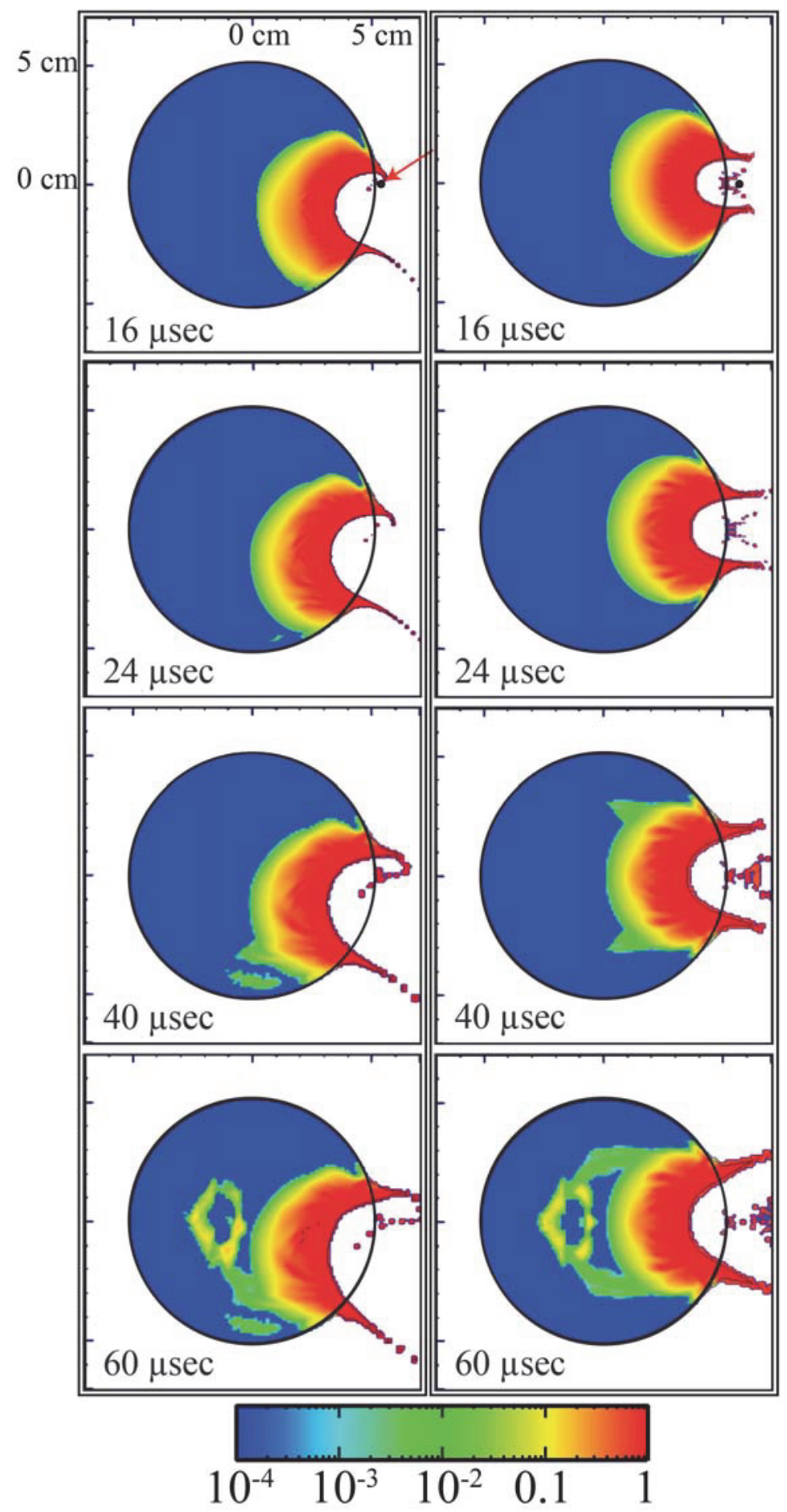

Figure 5. CTH model matched 1:1 for the impact shown in Figure 2 showing damage due to shear deformation. Impact conditions: $0.635-\mathrm{cm}$ Pyrex sphere impacting at an angle of $47^{\circ}$ and $5.6 \mathrm{~km} / \mathrm{s}$. Times correspond to panels in Figure 1. $\mathrm{D}=0$ is undamaged material and $\mathrm{D}=1$ indicated failed material. Left: 2-dimensional slice through the center plane of the sphere parallel to the trajectory. Right: 2D slice perpendicular to the trajectory. Original sphere diameter and projectile size are shown for scale. The arrow indicates impact trajectory. 


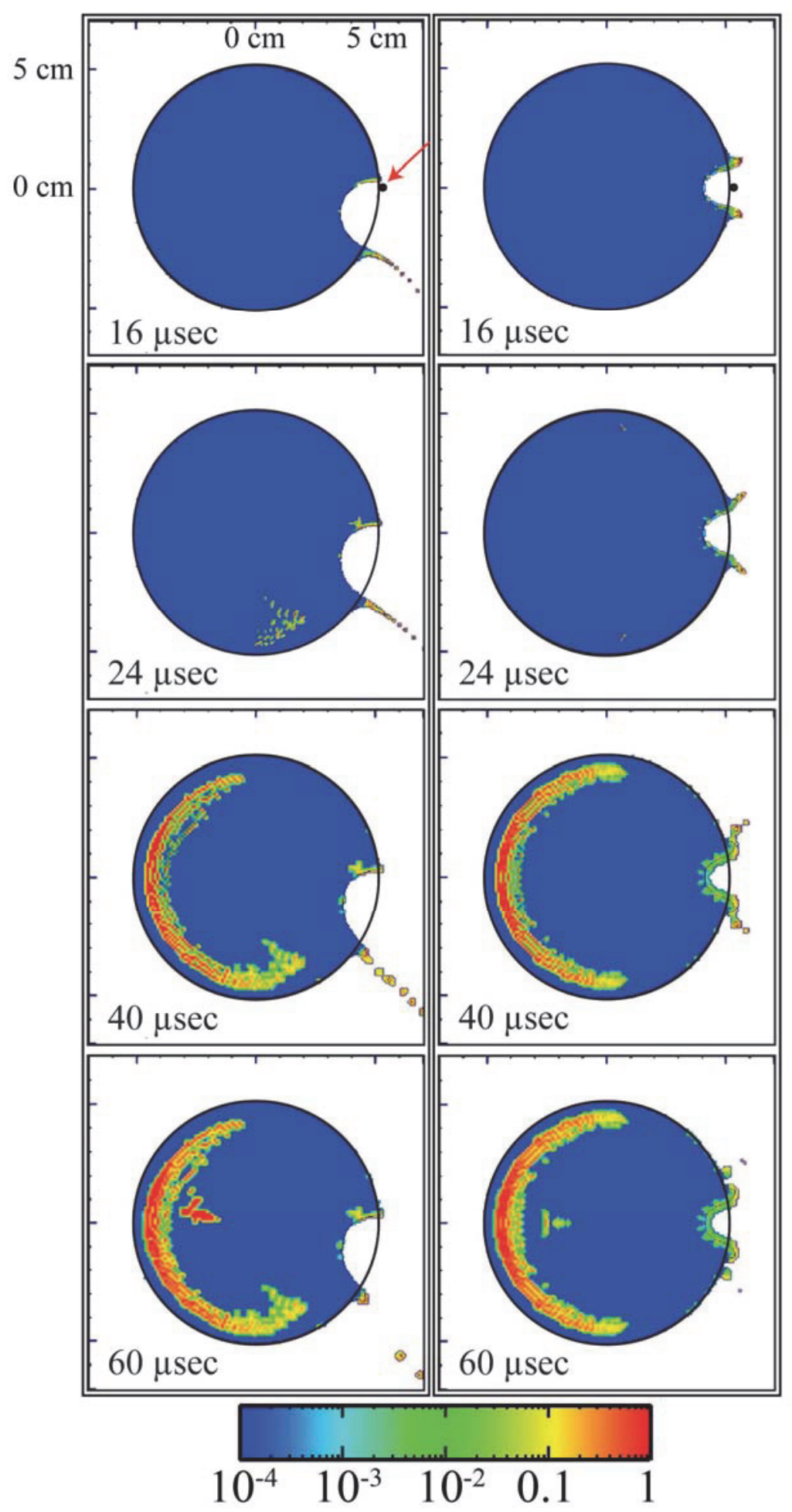

Figure 6. CTH model matched 1:1 for the impact shown in Figure 2 showing damage due to tensile failure (spallation). Impact conditions: $0.635-\mathrm{cm}$ Pyrex sphere impacting at an angle of $47^{\circ}$ and $5.6 \mathrm{~km} / \mathrm{s}$. Times correspond to panels in Figure 2. $\mathrm{D}=0$ is undamaged material, while $\mathrm{D}=1$ is failed material. Left: 2-dimensional slice through the center plane of the sphere parallel to the trajectory. Right: 2D slice perpendicular to the trajectory. The original sphere diameter and projectile size are shown for scale. The red arrow indicates the impact trajectory. 


\subsection{Laboratory-Scale CTH Results}

Impact conditions for the small-scale CTH simulations were identical to the experiments. The model corresponding to the experiment shown in Figure 2 was replicated for the shear failure (Figure 5) and the spall failure (Figure 6) criteria. The JCF damage parameter is used to illustrate regions of the target failing in shear (Figure 5). $\mathrm{D}=1$ indicates material that is completely failed (corresponding to opaque regions in the laboratory experiments), whereas values of $0<\mathrm{D}<1$ indicate damaged and weakened material. These figures show 2 -dimensional slices through the 3-dimensional model at the center planes of the sphere along the trajectory (left column) and parallel to the trajectory (right column).

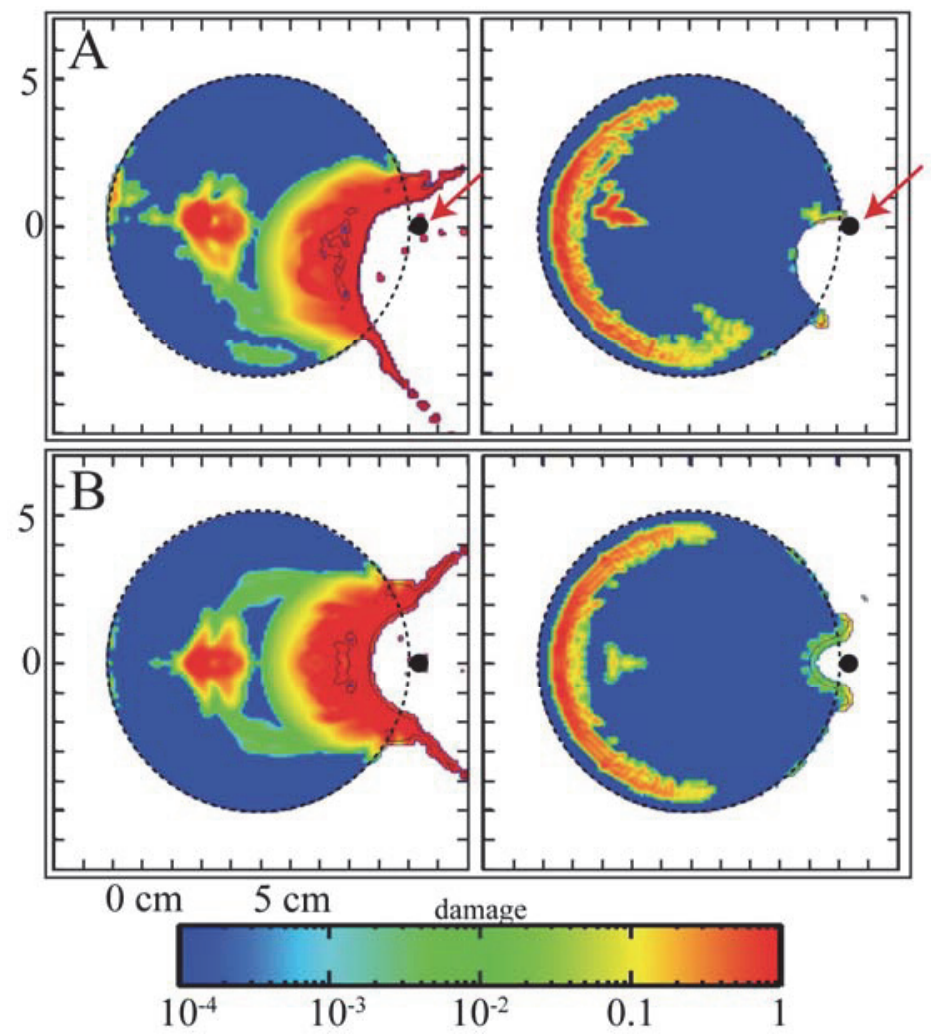

Figure 7. Damage structures $108 \mu \mathrm{sec}$ after impact. Conditions are the same as the experiment in Figure 1. A) Shear failure criterion (left) and spall failure criterion (right) from the side (parallel to the trajectory). B) Shear failure criterion (left) and spall failure criterion (right) along the trajectory. Note the central columnar damage zone in the spall criterion, whereas the damage planes align roughly perpendicular to the stalk in the shear failure criterion. The projectile size and original sphere cross-section are shown for scale. The red arrow indicates the impact trajectory.

Figures 5 and 6 illustrate the evolution of the damage zones, but it is also necessary to examine final damage structures using both failure criteria. Final damage patterns (time $=108$ $\mu$ sec after impact) are shown for this experiment in Figure 7 for the same two views shown in Figures 5 and 6. The near-surface failure seen in the spall criterion (right hand side of A and B) wraps around the sphere in the $3 \mathrm{D}$ target and is shown as a thin region here. Damage due to spallation overlain onto damage from shear damage then allows a comparison to the damage structures seen in the experiments (Figure 8). The 2D CTH slice reveals the subsurface failure 
extent more clearly than in the experiments where the haze near the surface obscures some of the deep subsurface failure.

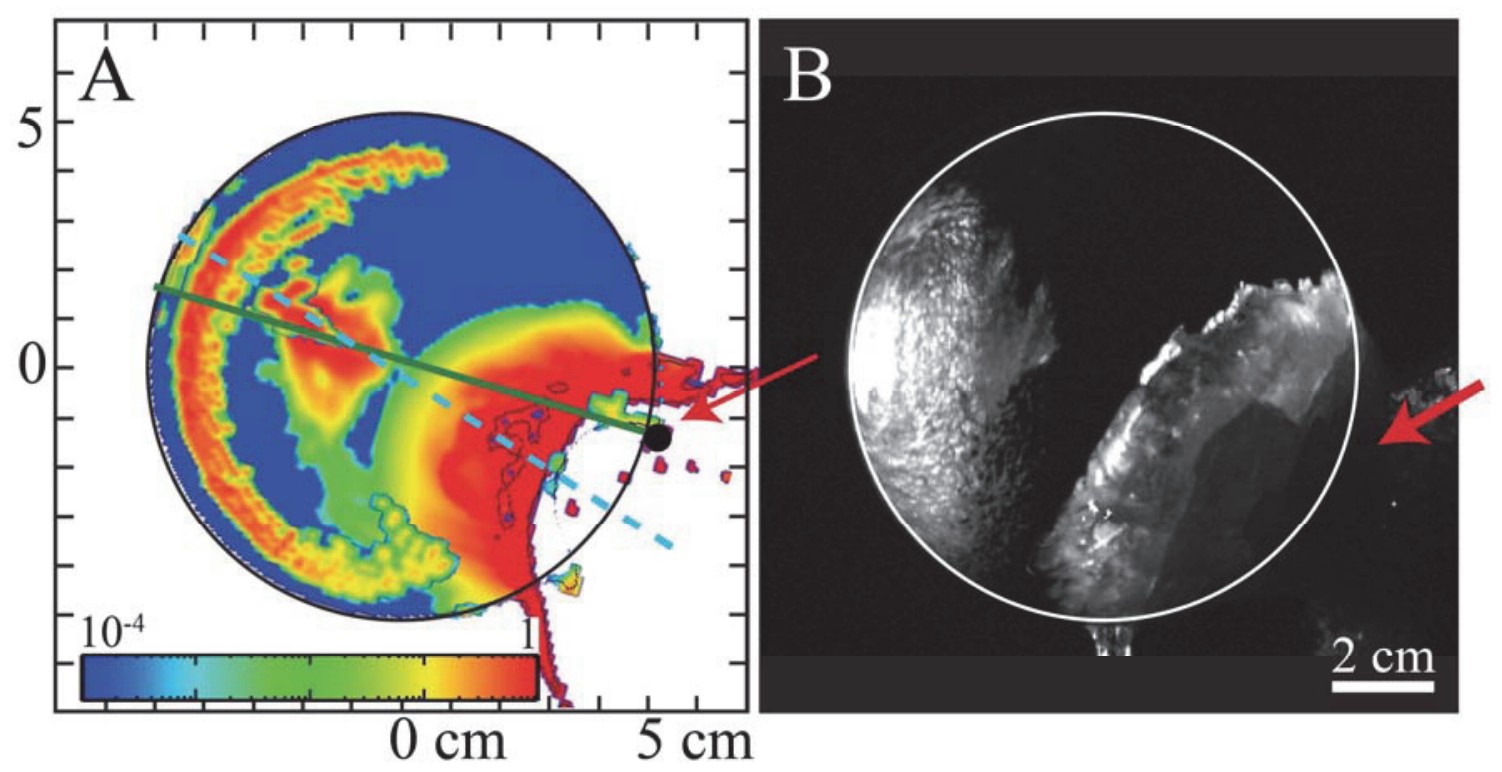

Figure 8. Damage structures $108 \mu \mathrm{sec}$ after impact. A) "Combined" failure criteria: spallation is overlain on shear failure; B) Corresponding experiment. Projectile size is shown for scale, the red arrow indicates impact trajectory, the light blue line shows the antipode to the crater center, and the green line indicates the antipode to the impact point.

Laboratory experiments reveal that varying the impact angle affects the orientation of the damage structures. Consequently, comparison models were run with identical impact conditions for each of the AVGR experiments. Figure 9 illustrates the final damage patters in two 2D slices from each impact condition: perpendicular to the trajectory (left) and parallel to the impact vector (right). The resulting evolution of the damage (Figure 9) remains similar to the $45^{\circ}$ reference set (Figures 5 and 6).

One of the advantages of numerical models is the ability to isolate different processes in detail. Tracking pressure throughout the impact event (Figure 10B, 10E) illustrates the shock wave traveling through the sphere as it interacts with the curved surface and reveals areas of the target are undergoing tensile or compressive stresses. These values can then be compared with other parameters, such as the magnitude of the shear stress (here, shown by a form of the second invariant of the deviatoric stress tensor, J2P; Figure 10A, 10D) or damage evolution (D, Figure $10 \mathrm{C}, 10 \mathrm{~F})$ in order to better understand failure processes and mechanisms within the target. The black and white dotted outlines in 10A and 10D show the outline of compressive (black) and extensional (white) regions overlain on top of the shear stress magnitude. The white lines in 10C and $10 \mathrm{~F}$, then, show regions of high shear stress overlain on damage. Here, the target has been rotated and the impact point is now at the top of the sphere. 

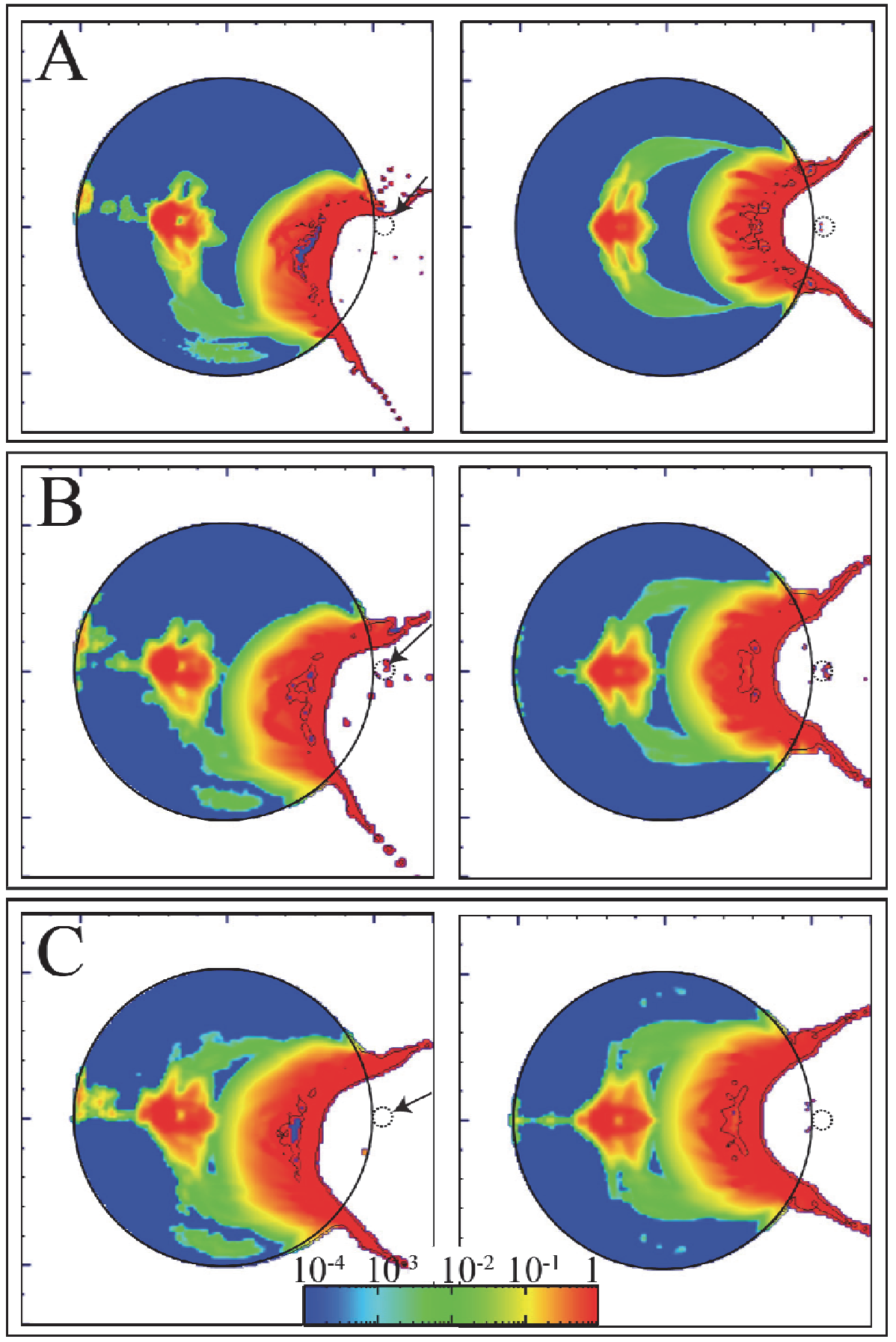

Figure 9. CTH models comparing to AVGR experiments of a 0.635-cm Pyrex sphere into 10-cm PMMA sphere. The damage parameter is shown for two cross-sections: parallel to the trajectory (left) and perpendicular to the trajectory (right) at $\sim 100 \mu \mathrm{sec}$ after impact. Projectile and original sphere size shown for scale. A) Impact angle: $40^{\circ}$, Impact velocity: $5.4 \mathrm{~km} / \mathrm{s}$. B) Impact angle: $47^{\circ}$, Impact velocity: $5.6 \mathrm{~km} / \mathrm{s}$; C) Impact angle: $65^{\circ}$, Impact velocity: $5.2 \mathrm{~km} / \mathrm{s}$. 


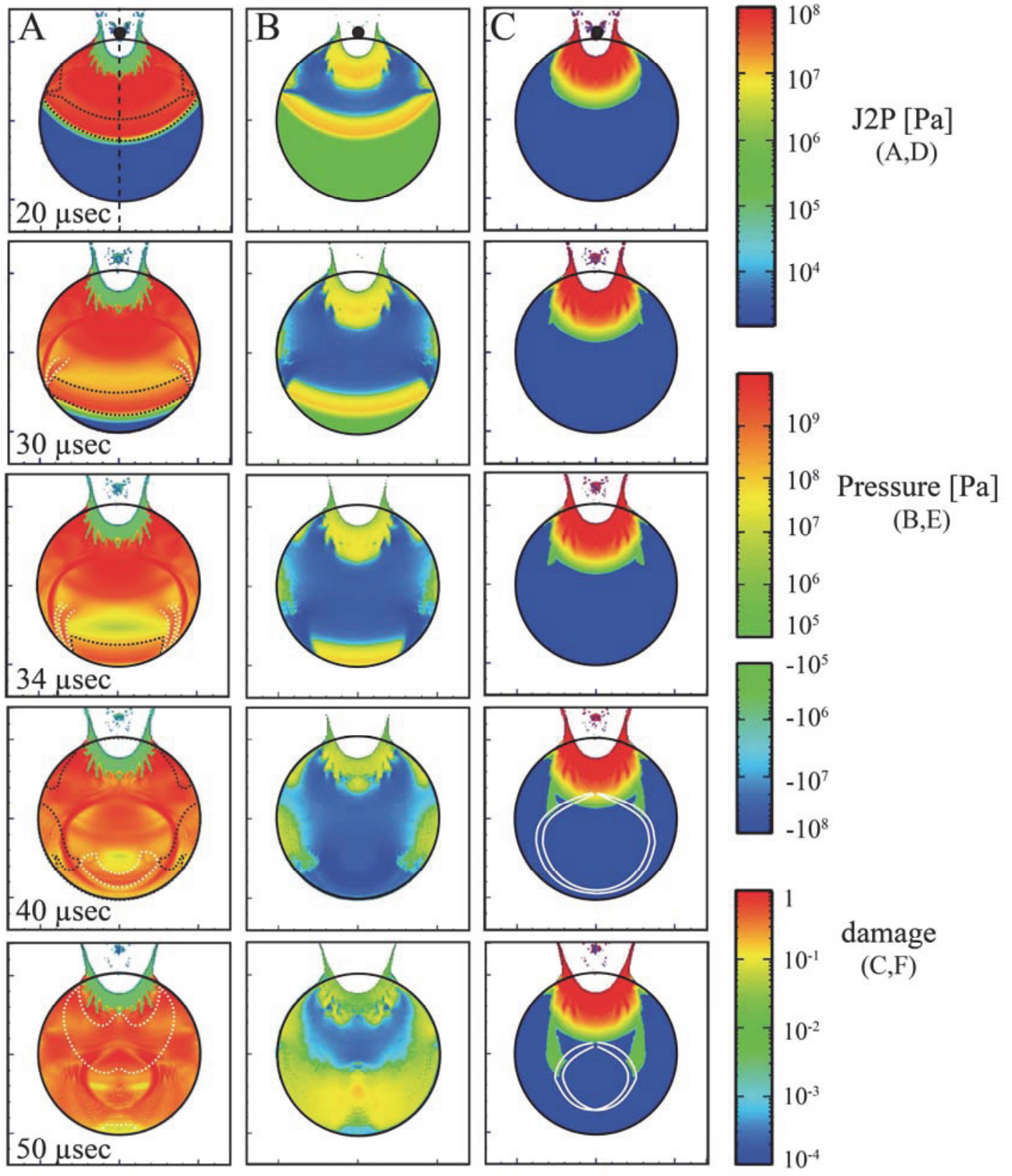

(Figure $10(a-c))$ 

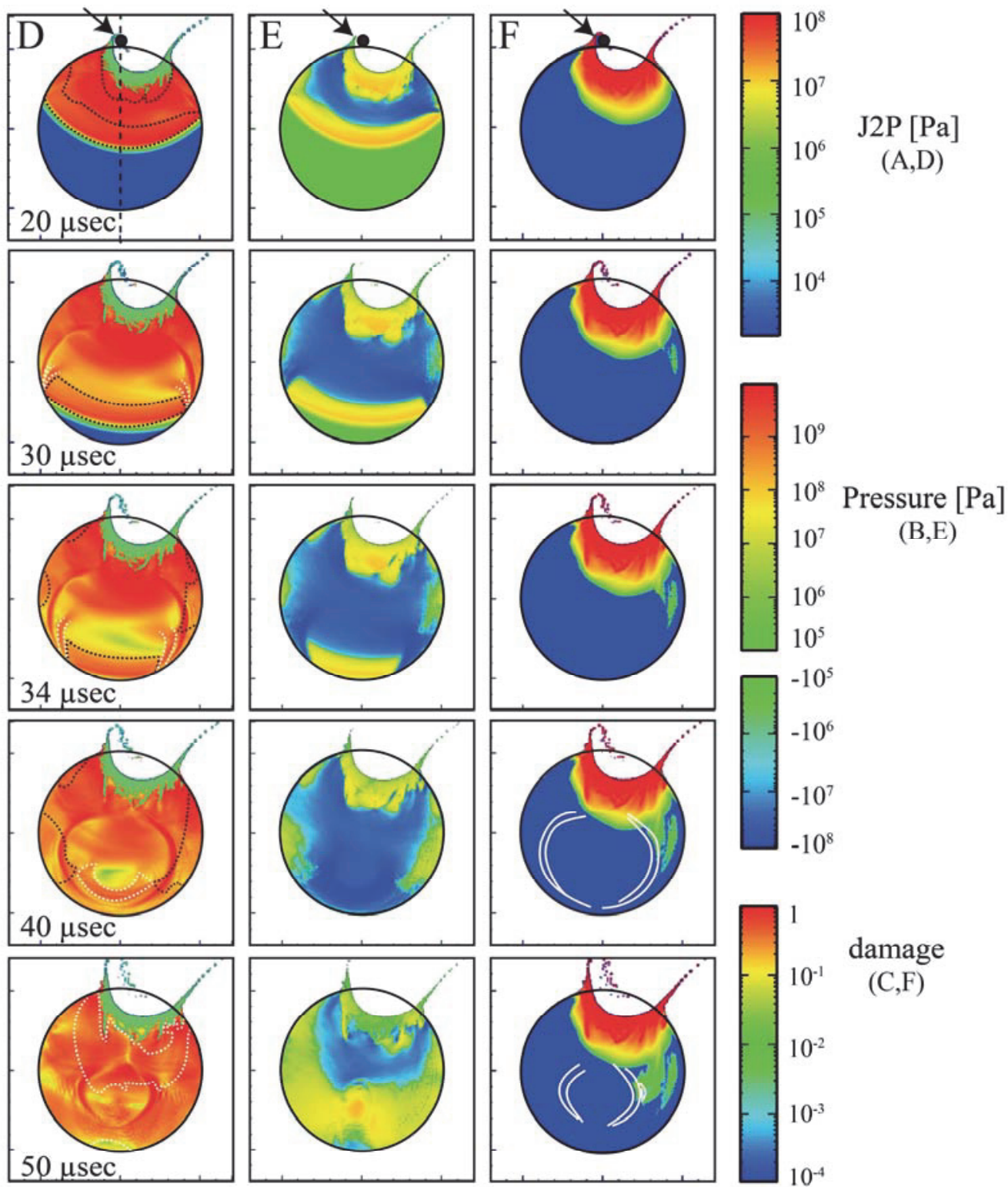

$10^{7} \quad(\mathrm{~B}, \mathrm{E})$

$10^{6}$

$10^{5}$

$-\begin{aligned} & -10^{5} \\ & -10^{6} \\ & -10^{7} \\ & -10^{8}\end{aligned}$

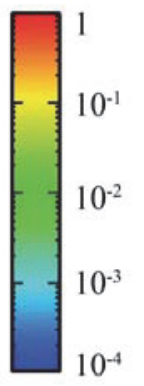

\section{damage}

$(\mathrm{C}, \mathrm{F})$

Figure 10. Shear stress and pressure through time following the impact of a $0.635-\mathrm{cm}$ Pyrex projectile into a $10-\mathrm{cm}$ PMMA sphere at $40^{\circ}$ and $5.4 \mathrm{~km} / \mathrm{s}$. Horizontal rows are all at the same time after impact, which is noted in the left-hand column. Images are 2D slices through the 3D model, and the planes go through the center plane of the sphere (location indicated by black dotted lines in A and D). Frames A, B, and C are perpendicular to the trajectory, whereas D, E, and $\mathrm{F}$ are parallel to the trajectory; projectile size is shown for scale. The material apparently suspended within the crater in frames A-C is actually part of the ejecta that has folded over, which can be seen in D-F. Frames A and D show the magnitude of the shear stress (J2P). Frames $\mathrm{B}$ and $\mathrm{E}$ show pressure through time. Frames $\mathrm{C}$ and $\mathrm{F}$ show the damage parameter, $D$. Black and white curves outline regions in tension (white) and compression (black) from B and E: positive values indicate compression, and negative values indicate tension. White curves in $\mathrm{C}$ and $\mathrm{F}$ outline regions with the highest values of J2P, which indicates that damage forms and grows in regions of high shear stress. 


\subsection{Large-Scale CTH Results}

CTH models simulating an impact into asteroid 4 Vesta reveal details about possible stress states causing internal damage at much larger scales. During the first $60 \mathrm{sec}$ after impact (the time for the shock wave to cross the body once; corresponding to $\sim 30 \mu \mathrm{sec}$ in laboratory experiments), the subsurface experiences extensive shear stress (Figure 11). In the next several hundred seconds, the stress state becomes more complicated, and large regions of the subsurface undergo extreme tensile stresses in addition to shear stress (Figure 12). The regions of maximum tensile stress are located downrange and opposite of the impact point, resulting in an asymmetric damage pattern within the body, similar to what is seen in laboratory experiments. Material on either side of the crater will likely delaminate and leave behind exposed mantle material, and a large portion of the asteroid downrange of the impact point will be fractured. Lastly, Figure 12 reveals a large region near the core mantle boundary will fail and fracture in deep, sub-parallel failure planes. While different in detail from the small laboratory experiments, the inferred sequence and style of failure is intriguingly similar.

\section{Discussion}

\subsection{Laboratory Scale Processes}

Laboratory experiments of oblique impacts reveal that deep, antipodal damage is not centered on the antipode to the crater center, but rather the point of first contact (Figure 4). The antipodal damage is separated into two main regions: the center column of damage pointing back towards the impact point, and the planes perpendicular to the central damage column (Figures 2, $7,8)$. These two main regions result from different processes. The central column of damage is due to tensile failure as shock waves coalesce and concentrate at the farside of the target [Rinehart, 1960] (Figure 6, 7, and 10). The planes, however, are formed by shear stresses set up behind the shock wave (Figures 5-8, and 10), similar to the blades observed in oblique impacts into PMMA blocks [Stickle and Schultz, 2010; Stickle and Schultz, 2011; Stickle and Schultz 2014]. Regardless of impact angle, the interior failure planes begin to form around $50 \mu \mathrm{sec}$ after impact (Figure 2, 5), when shear stresses are highest near the middle of the sphere (Figure 10). Further, the region of failure is under compression, inhibiting tensile fracturing. 

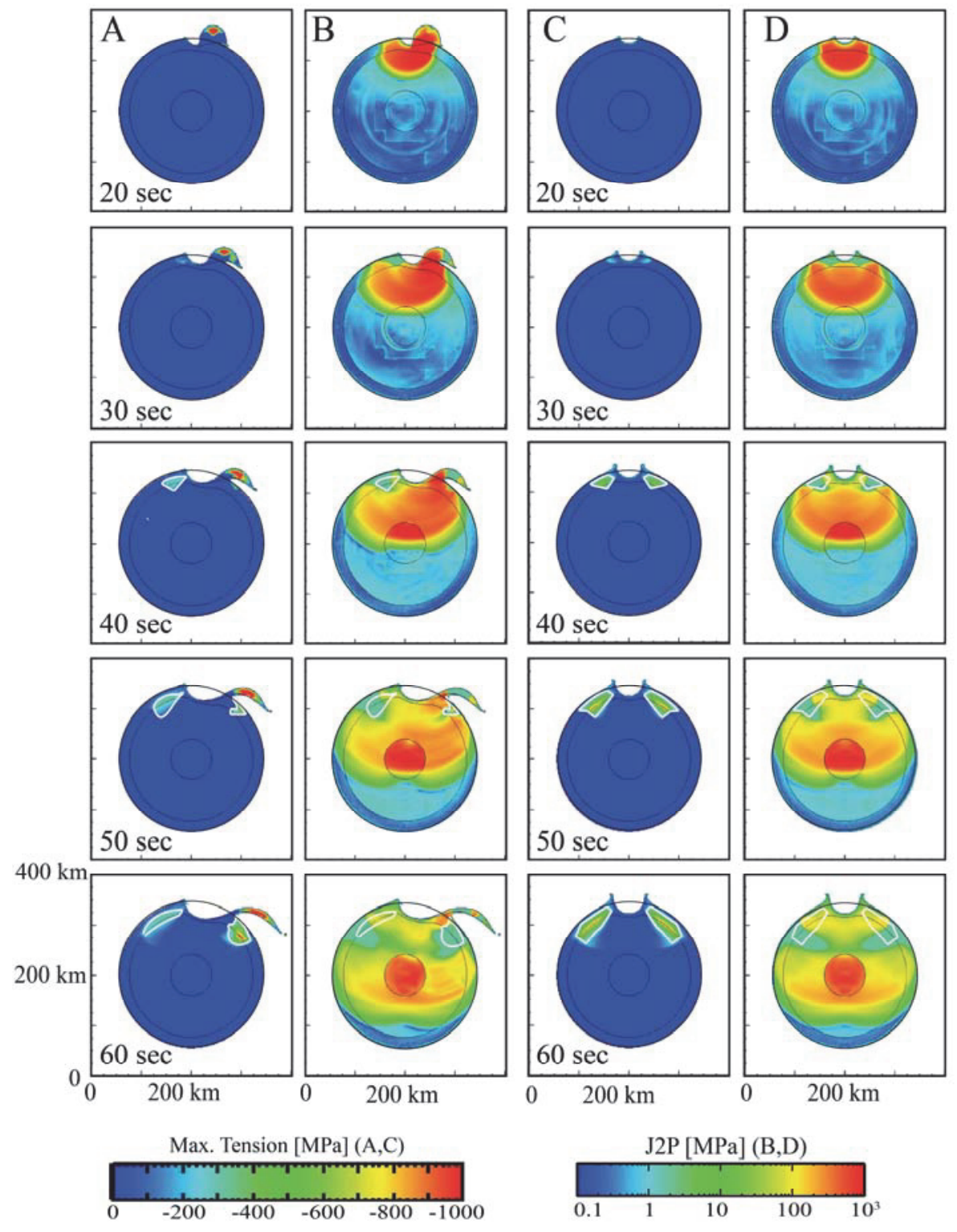

Figure 11. Shear stress and pressure for the first $100 \mathrm{sec}$ after impact of a 50-km dunite projectile into the asteroid Vesta. The 578-km diameter asteroid has a metallic core $(164 \mathrm{~km})$, a dunite mantle (165 km thick) and a basaltic crust (42 km thick) [after Ruzicka et al., 1997]. The horizontal rows are all at the same times, and the columns represent slices through the asteroid. $(A, B) 2 D$ slice parallel to the trajectory in the center plane of the sphere, $(C, D)$ : 2D slice perpendicular to the trajectory in the plane of the impact point. $(\mathrm{A}, \mathrm{C})$ show the maximum tensile stress experienced by the target throughout the evolution; $(\mathrm{B}, \mathrm{D})$ show the magnitude of the shear stress (represented by the second invariant of the deviatoric stress tensor, J2P). The mantle is considered fractured when local stress exceeds the overburden by $180 \mathrm{MPa}$ [Cohn and Ahrens, 1981 (this occurs at the transition from dark blue to cyan). 

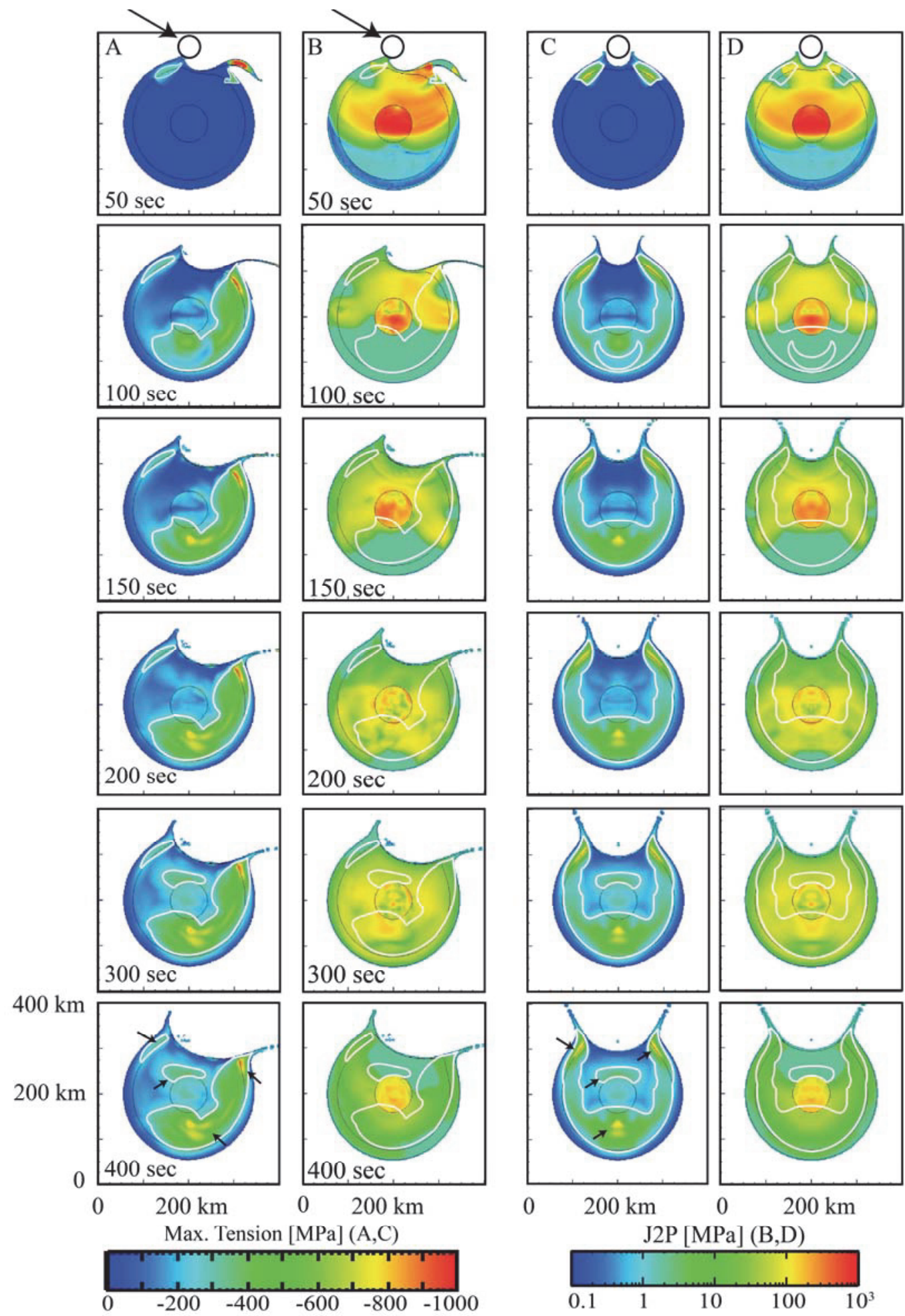

Figure 12. The same parameters as in Figure 11, revealing the later evolution of subsurface damage. The black arrows in $\mathrm{A}$ and $\mathrm{C}$ indicate regions of failure. 
Impact angle also affects the orientation of the rosette feature (Figure 4, 9). In general, the central column is offset from the crater center antipode and the orientation angle steepens for increasingly oblique impacts (Figure 4). Similarly, the angle between the extending planes and the normal to the line connecting the impact point to its antipode also depends on impact angle (Figure 13 and Table 2). The orientation of the failure planes becomes less orthogonal to the normal connecting the impact point and its antipode as impact angle decreases.

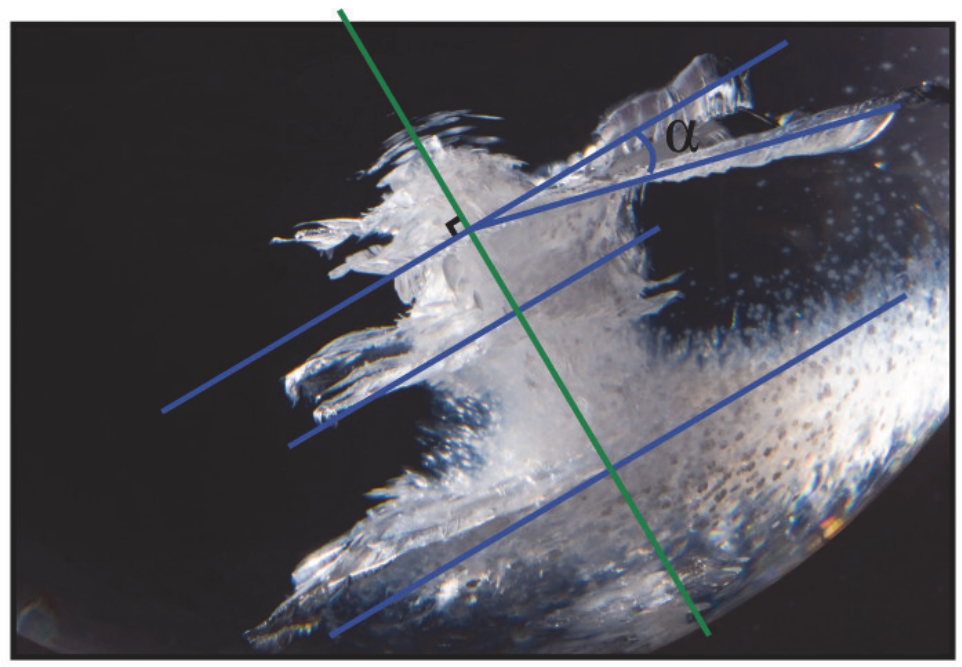

Figure 13. Schematic showing how the angle $\alpha$ is defined: the angle between the normal to the line connecting the impact point and its antipode (purple) and the orientation of the "petal" failure planes.

Table 2. Orientation of damage structures measured for three different laboratory experiments

\begin{tabular}{cccc}
$\begin{array}{c}\text { Impact angle } \\
\text { [deg] }\end{array}$ & $\begin{array}{c}\text { Number of } \\
\text { "petals" }\end{array}$ & $\begin{array}{c}\text { Angle to normal }(\boldsymbol{\alpha}) \\
{[\mathrm{deg}]}\end{array}$ & $\begin{array}{c}\text { Angle between } \\
\text { impact point and } \\
\text { crater center } \\
\text { antipodes [deg] }\end{array}$ \\
\hline 40 & 3 & $\alpha 1=19$ & 13 \\
& $\alpha 2=12$ & \\
& $\alpha 3=7.6$ & 13 \\
47 & 3 & $\alpha 1=15-16$ & \\
& & $\alpha 2=14-15$ & 5 \\
& & $\alpha 3=12$ & \\
& & $\alpha 1=13$ & \\
\hline
\end{tabular}

The near-surface, hazy region enveloping the downrange portion of the sphere represents incipient spallation. Laboratory experiments examining catastrophic disruption resulted in entire layers of basaltic and glass spheres spalling from small, spherical targets [e.g., Gault and Wedekind, 1969; Nakamura and Fujiwara, 1991; Nakamura et al., 1994]. The three experiments in this study did not experience that level of disruption, however cracks are present at the antipode to the impact point, suggesting that material may have spalled from the farside of the 
target for slightly higher impact energy. The haze may therefore represent the beginning stages of large-scale spallation and disruption of the target. Conversely, glass spheres did not develop the interior tiered rosette pattern, due to relief in strain caused by spallation or the nature of amorphous glass [Schultz and Gault, 1975].

\subsection{Vesta from Dawn}

The Dawn mission recently left the giant asteroid 4 Vesta. The goals of the mission are to characterize the conditions and processes of the earliest epoch in the solar system by studying two of the largest asteroids (protoplanets) remaining in the asteroid belt [Russell et al., 2007]. Earth and Hubble Space Telescope-based observations provided first-order views of Vesta, both indicating that Vesta has a basaltic crust indicative of melting and extensive thermal evolution, and is a dry, differentiated body [McCord et al., 1970]. Further, the reflectance spectrum matches the composition of the HED meteorites [e.g., McFadden et al., 1977; Feierberg and Drake, 1980; Feierberg et al., 1980; Gaffey, 1997], and dynamical models indicate that the HED meteorites (and the Vesta dynamical family) likely originated from Vesta [Williams 1989; Zappalá et al., 1995; Binzel and Xu, 1993; Burbine et al., 2001]. The asteroid is an oblate spheroid, with radii of 286.3-km, 278.6-km and 223.2- $\mathrm{km} \pm 0.1 \mathrm{~km}$ [Russell et al. 2012], and it has two large craters on the south pole [Thomas et al., 1997; ]: the $500 \pm 20-\mathrm{km}$ Rheasilvia basin overprinting the older, $400 \pm 20$-km Veneneia basin [Jaumann et al., 2012; Schenk et al., 2012]. The mass of Vesta is $2.596076 \pm 0.00001 \times 10^{20} \mathrm{~kg}$ with a bulk density of $3456 \mathrm{~kg} / \mathrm{m}^{3} \pm 1 \%$ [Russel et al., 2012], and a core size up to $25 \%$ by mass [Dreibus et al., 1997; Ruzicka et al., 1997; Righter and Drake, 1997]; models of Vesta's interior structure indicate the core is likely 107-113 km [Russel et al., 2012].
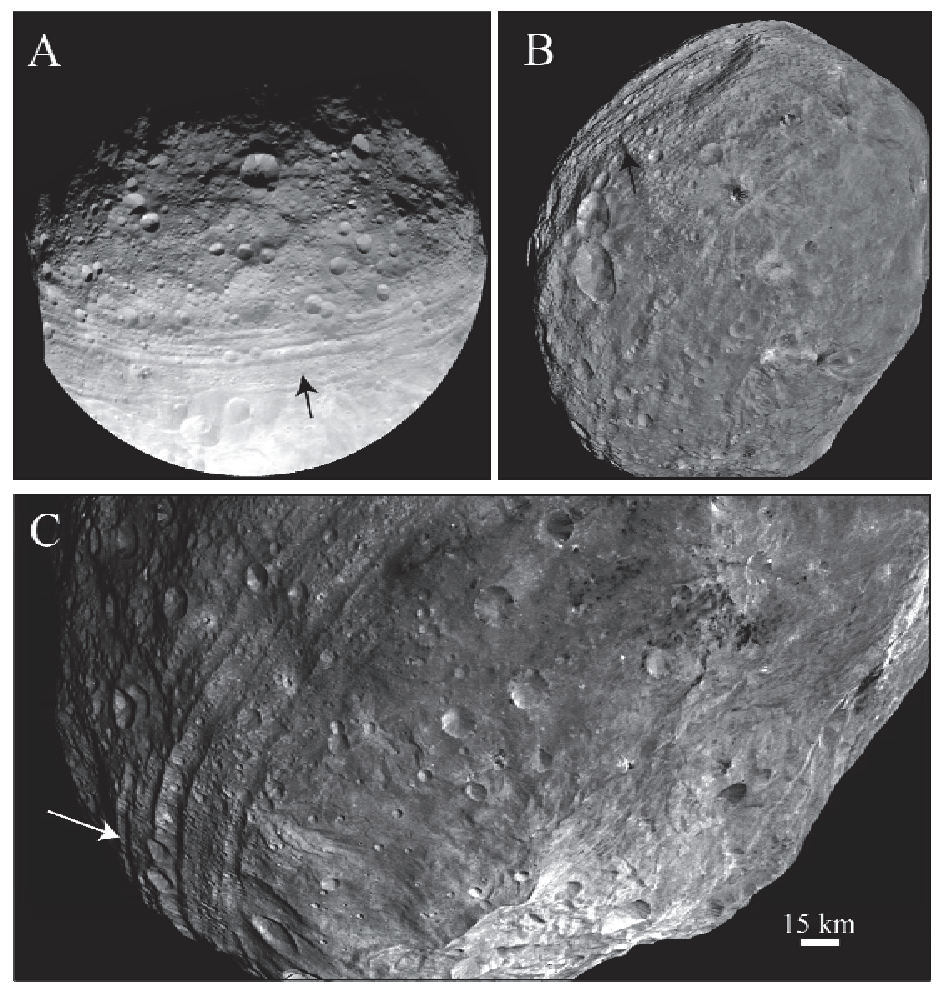

Figure 14. Images of the grooves on Vesta from the Dawn spacecraft. A) Image of the full asteroid, taken at a distance of 5,200 km, showing equatorial troughs (black arrow); B) Image of 
Vesta showing troughs near the north pole (black arrow). The image was taken by the Dawn spacecraft at a distance of $5,200 \mathrm{~km}$. Resolution is $\sim 500 \mathrm{~m} /$ pixel; C) Close-up view of the morphology of Vesta's equatorial troughs. The central mound of Rheasilvia is seen in the bottom right of the image. Image credits: NASA/JPL-CalTech/UCA/MPS/DLR/IDA.

Images returned from the Dawn spacecraft show two sets of troughs on Vesta's surface (Figure 14, 15): one set parallels the equator, and the other is seen nearer to the north pole [Le Corre et al., 2012; Jaumann et al., 2012; Schenk et al., 2012a,b; Scully et al., 2012]. The equatorial trough set contains 85 linear structures with lengths varying from 19-380 km and widths up to $15 \mathrm{~km}$. The longitudes of the poles to these structures vary, but seem to circle the central mound of the large south-pole impact basin Rheasilvia [Buczkowski et al., 2011; Buczkowski et al., 2012; Jaumann et al., 2012]. Nearer to the north pole, the primary structure is $390 \mathrm{~km}$ long and $38 \mathrm{~km}$ wide. There are currently 7 mapped linear structures in this set, with lengths from $31-212 \mathrm{~km}$, and the poles to these structures cluster near the center of Veneneia, the second, smaller impact basin near the south pole [Buczkowski et al., 2011; Buczkowski et al., 2012]. The two sets of troughs crosscut each other near the equator at approximately $300-330^{\circ}$ $\mathrm{W}$ longitude, and they meet at an angle of $30^{\circ}$. Based on the crosscutting relationships, the equatorial troughs are younger than those near the north pole. Further, the equatorial troughs also seem to be more well-preserved than the majority of Vesta's surface, indicating that they formed after the north polar troughs [Buczkowski et al., 2011]. Alternatively, the troughs may be older sets of faults that have been re-activated since their formation (following [Asphaug, 1996]), making a precise age relationship difficult to determine.

Large-scale CTH models in conjunction with the insights gained from laboratory experiments provides new clues into the formation of the trough sets on Vesta. Comparing the small-scale CTH simulations to laboratory experiments provided a better understanding of the failure processes following impact. Hydrocodes represent one way to scale processes seen in laboratory experiments to features observed on planetary surfaces. Here, the small-scale comparisons performed first indicated regions of the model that are a good match to experiments, providing intuition into what processes can be easily scaled with further, large-scale models. Examining similar processes in the large-scale models to those that were seen in the small-scale models gives confidence that we are capturing real phenomena.

The large-scale models allow examination of fracture and failure deep within Vesta. The upper limit for dynamic tensile strength of basalt is $180 \mathrm{MPa}$ [Cohn and Ahrens, 1981], whereas the lithostatic pressure at the core-mantle boundary is $130 \mathrm{MPa}$ (Figure 1). Material is considered fractured when stresses exceed the lithostatic load (the overpressure) by $180 \mathrm{MPa}$, which gives a maximum strength (for any depth within Vesta) of $\sim 310 \mathrm{MPa}$. We expect, then, that material within the body will become damaged and fracture around $200 \mathrm{MPa}$ corresponding to the transition from dark blue to cyan in the maximum tensile stress diagrams (Figures 11 and 12). Consequently, a large region of the asteroid should have been subjected to tensile stresses great enough for fracture and failure (Figures 11 and 12). Just as in the laboratory experiments, shear processes are also likely important in subsurface evolution (Figure 12). The white outlines in Figure 12B and 12D indicate the regions where tensile stresses are large enough to cause fracturing. These regions overlap with, or form directly before, regions of high shear stress. Temporally, the combination of these two stress states suggests that the subsurface of Vesta may be damaged or fractured due to tensile stresses, but then fail and slide due to high shear stresses 
behind the shock wave. This pattern is seen even to late times (Figure 12), as the shock and rarefaction wave reflect and coalesce throughout the body.

The location of subsurface failure regions within Vesta suggests a possible formation scenario for the troughs seen on the surface. It is known that seismic stresses can be enhanced at the antipode to large impacts, which can result in increased damage formation [e.g., Schultz and Gault, 1975; Asphaug et al., 1996; Schultz and Crawford, 2011; Meschede et al., 2011]. Numerical studies by Asphaug et al. [1996] and Benz and Asphaug [1994] reveal that the grooves (troughs) in Pola Regio, a region of the asteroid Ida, and the grooves on Phobos may have formed by large impacts [Fujiwara and Asada, 1983; Asphaug and Melosh, 1993]. Smoothed-particle Hydrodynamics (SPH) simulations show that far-field stress waves will focus and cause local damage in the subsurface of Ida, which may then be reactivated by later impacts [Asphaug et al., 1996]. The CTH calculations shown here reveal that similar processes may have occurred on Vesta.

In contrast with previous studies on asteroid damage, the large-scale simulations here suggest that the deep failure within the body may be due to mixed-mode processes and not purely a result of tensile stresses. The combination of high shear stress magnitudes overprinting weakened or pre-damaged material lasts for hundreds of seconds (Figure 12). A typical earthquake rupture on Earth propagates at approximately $3 \mathrm{~km} / \mathrm{s}$ [Stein and Wysession, 2003]. Assuming: 1) a similar propagation rate for Vesta, and 2) continued stress loading will cause the deep fractures to grow to the surface, fractures forming deep within the body will reach the surface after approximate 100-150 seconds. During this time period, damaged material is continually subjected to high shear stresses, which creates localized shear planes.
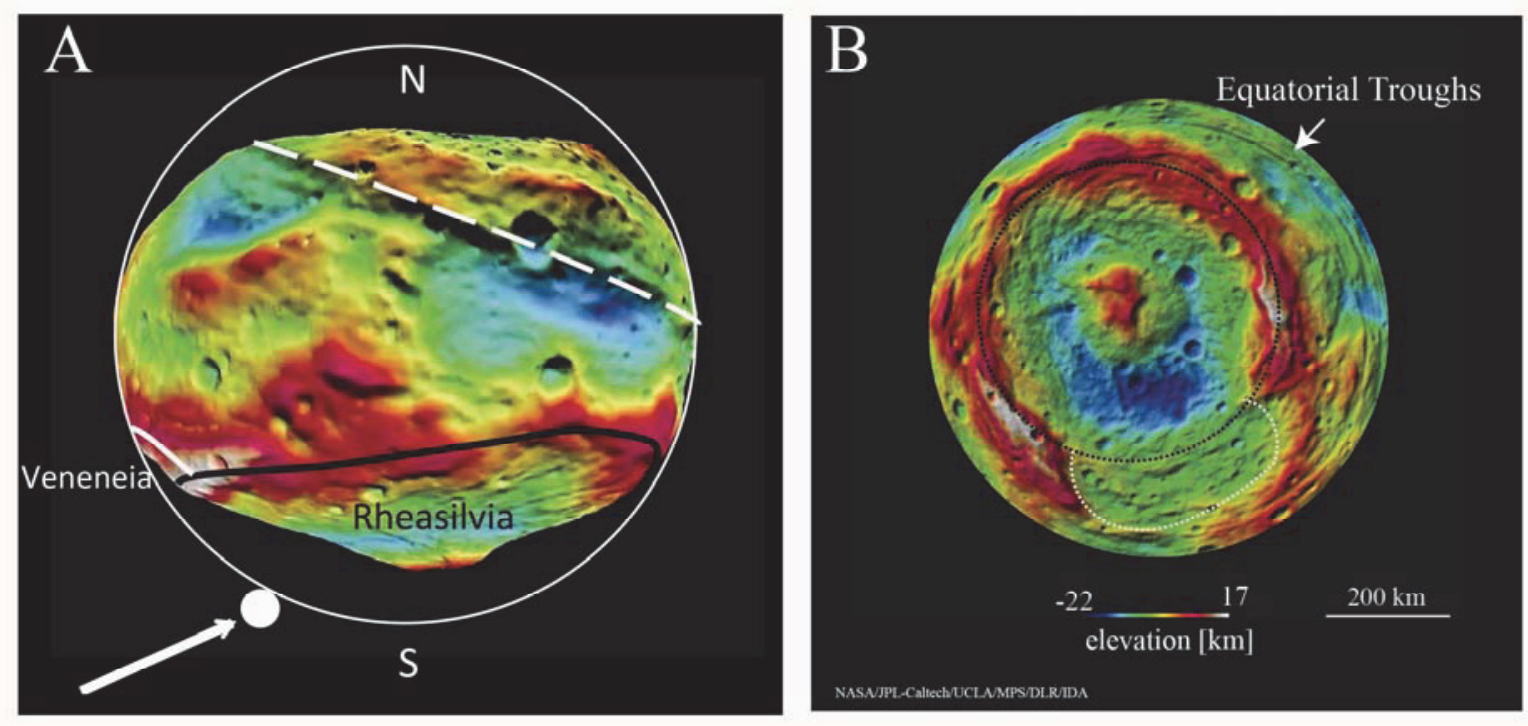

Figure 15. A) Schematic of impact point and oblique trajectory for projectile resulting in the Rheasilvia Basin. The N-polar troughs are obtuse to Rheasilvia and Veneneia in the same manner as the subsurface failure planes in the experiments. B) Elevation map of Vesta's south pole, showing the large impact crater Rheasilvia (outlined in black) and a portion of the Veneneian rim (outlined in white). Rheasilvia has a diameter of $\sim 500 \mathrm{~km}$ (80\% of the asteroid) and partially obscures the older Veneneia basin. The central mound is approximate $20-25 \mathrm{~km}$ high and $180 \mathrm{~km}$ wide [Schenk et al., 2012]. White arrows locate the equatorial troughs, which 
are centered around a point offset from the Rheasilvia basin center [Jaumann et al., 2012]. Image credit: NASA/JPL-Caltech/UCLA/MPS/DLA/IDA.

Observations of impacts on Vesta combined with laboratory experiments also provide insight in other critical impact parameters. The south pole of Vesta is covered by two large impact basins (Rheasilvia and Veneneia) with a diameter approaching that of Vesta (Figure 15). Rheasilvia has an elevation range of $\sim 40 \mathrm{~km}$, and contains a central mound. If the subsurface shear planes observed in the laboratory are small-scale analogs for the trough features observed on Vesta, then the orientation of the damage region might indicate the impact location and angle by the angle of offset from the impact crater center antipode. Table 2 shows that, for laboratory experiments, the angle between the antipode to the impact point and the antipode to the crater center decreases from $\sim 13^{\circ}$ to $5^{\circ}$ as impact angle increases from $40^{\circ}-65^{\circ}$. This variation is consistent with results from simulations of the South-Pole-Aitken Basin formation on the lunar surface [Schultz and Crawford, 2011]. As a first cut, we can apply this logic to Vesta as well. By measuring the angle of the trough offset from the basin center antipode, we can place some constraints on the impact point antipode and thus likely impact point. Assuming impact angle affects angle of offset for troughs on Vesta similarly to what was seen in experiments, we see that the Rheasilvia impactor likely collided at an angle less than $40^{\circ}$ with respect to the surface tangent (Figure 15, 16). Impact angles higher than $40^{\circ}$ result in improbable impact points too near the crater center (Figure 16B and 16D) to create the morphology that is seen on Vesta now. Further study will be done to fully characterize the variation with impact angle such that more stringent constraints can be placed on the impact location and trajectory. 

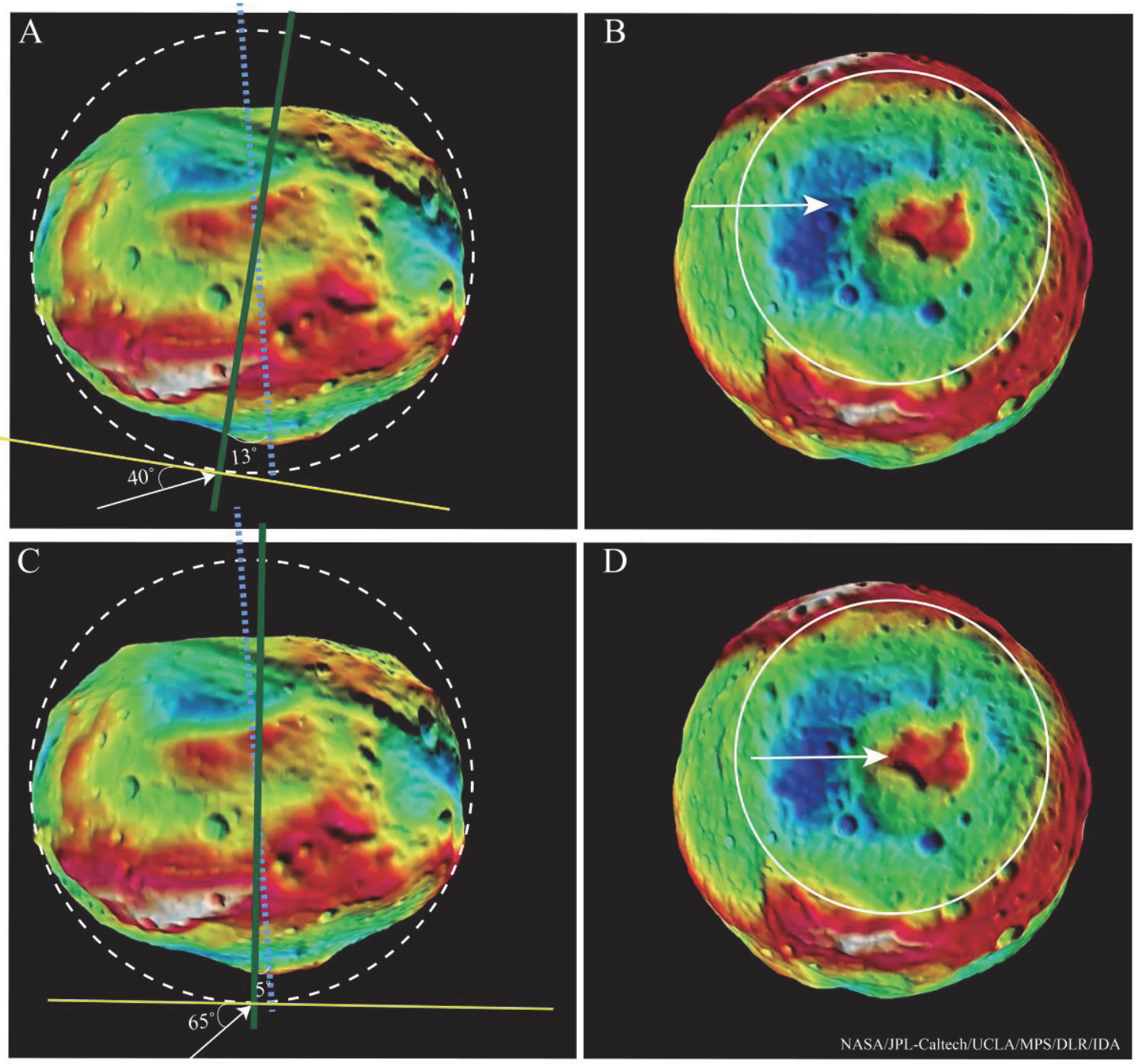

Figure 16. Inferred impact points at two different impact angles based the angle between the center of the crater structure and the impact point as measured in laboratory experiments. The white dashed line indicates an assumed spherical shape for Vesta with a $289-\mathrm{km}$ radius. The blue lines point to the crater center antipode, and the green lines show the impact point and its antipode. A) Inferred impact point for $40^{\circ}$ impact; B) view of the south pole of Vesta showing inferred impact point from A. Direction and impact point indicated by the white arrow; C) Inferred impact point for a $65^{\circ}$ impact shown by the tip of the arrow; D) View of the south pole of Vesta showing the estimated impact point from panel C. Images were taken from an animation showing the surface structure of Vesta as observed by the Dawn spacecraft. Image credit: NASA/JPL-Caltech/UCLA/MPS/DLA/IDA.

\section{Conclusions}

Laboratory experiments coupled with three-dimensional CTH models reveal details about subsurface failure within spherical targets. Laboratory experiments are characterized by three main damage regions: the intensely fractured and spalled region surrounding the impact point; central and antipodal failure plains (the "rosette"); and shallow, near-surface incipient spallation and shear downrange from the impact point. Rarefaction waves reflected off the free surface 
converge inside the sphere centered on the antipode to the first contact (offset from the crater center) and create a damage column. Numerical simulations reveal that deep failure planes result from shear deformation following passage of the shock wave. The orientation of the planar damage features, the number of sub-parallel failure planes, and the location and extent of shallow hazy failure depend on impact angle.

Insight gained from these laboratory and small-scale numerical experiments prompts a new interpretation of surface features observed on asteroids. Sets of linear troughs observed on the asteroid 4 Vesta represent just one example, but these interpretations could apply to similar features occurring on other small bodies. Three-dimensional CTH calculations of large, oblique impacts onto a differentiated Vesta induce damage over large regions of the subsurface. Such simulations confirm that the linear features may be surface expressions of subsurface failure and faulting. A combination of tensile damage and high values of shear stress indicate that these features may be the result of shear localization in subsurface failure planes similar to what is seen in laboratory experiments. Comparison between the orientation of damage structures in the laboratory and failure regions within Vesta may then be used to constrain impact parameters. The combination of experiments and models presented here suggests that the Rheasilvia basin was formed by an impactor colliding with Vesta at $\sim 5 \mathrm{~km} / \mathrm{s}$ and an angle less than $40^{\circ}$. Future detailed structural studies of the surface troughs on Vesta provide a means to test these hypotheses.

\section{Acknowledgements}

AMS was funded by the NASA Planetary Geology and Geophysics program, grant NNX 08AM45G, for this research. AMS thanks Amy Barr, Karen Fischer, Greg Hirth, and John Spray for helpful reviews that strengthened the manuscript. D.A. Crawford acknowledges that Sandia is a multi-program laboratory operated by Sandia Corporation, a Lockheed Martin Company, for the United States Department of Energy under Contract DE-AC04-94AL85000. The authors would also like to thank two anonymous reviewers for their comments to help finalize this manuscript.

\section{References}

Amsden, A.A., H.M. Ruppel, and C.W. Hurt (1980), SALE: A simplified ALE computer program for fluid flow at all speeds. Technical Report LA-8095, Los Alamos National Laboratory.

Asphaug, E. (1997), Impact origin of the Vesta family, Meteoritics \& Planetary Science, 32(6), 965-980.

Asphaug, E., and H.J Melosh (1993), The Stickney impact of Phobos: a dynamical model. Icarus, 101, 144-164.

Asphaug, E., J.M. Moore, D. Morrison, W. Benz, M.C. Nolan, and R.J Sullivan (1996), Mechanical and Geolgoical Effects of Impact Cratering on Ida, Icarus 120, 158_184.

Barnes, J.E., and P. Hut (1986), A hierarchicial I(N Log N) force calculation algorithm, Nature, 324, pp. 446-449.

Benz, W., and E. Asphaug (1994), Impact Simulations with Fracture. I. Method and Tests, Icarus, 107(1), 98-116.

Benz, W., and E. Asphaug (1999), Catastrophic Disruptions Revisited, Icarus, 142(1), 5-20. 
Bills, B.G., S.W. Asmar, A.S. Konopliv, R.S. Park, and C.A. Raymond (2014) Harmonic and statistical analyses of the gravity and topography of Vesta, Icarus, in press. doi: 10.1016/j.icarus.2014.05.033.

Binzel, R.P, and S. Xu (1993) Chips off of asteroid 4 Vesta: Evidence for the parent body of basaltic achondrite meteorites. Science, 260, 186-191.

Binzel, R. P., M. J. Gaffey, P. C. Thomas, B. H. Zellner, A. D. Storrs, and E. N. Wells (1997), Geologic mapping of Vesta from 1994 Hubble Space Telescope images, Icarus 128(1), 95103.

Bottke, W.F., A. Cellino, P. Paolicchi, and R.P. Binzel (2002), An overview of the Asteroids: The Asteroids III Perspective, in: Asteroids III (eds. W.F. Bottke, A. Cellina, P Paolicchi, and R.P. Binzel). The University of Arizona Press: Tuscon, AZ., pp. 3-17.

Bowling, T.J. et al. (2013a), Formation of Equatorial Graben Following the Rheasilvia Impact on Asteroid 4 Vesta, LPS XLIV, Abstrat\#1673.

Bowling, T.J. et al. (2013b), Antipodal terrains created by the Rheasilvia basin forming impact on asteroid 4 Vesa, Journal of Geophysical Research Planets, 118, 1-14, doi: 10.1002/jgre.20123.

Bruesch, L.S., and E. Asphaug (2004), Modeling global impact effects on middle-sized icy bodies: applications to Saturn's moons, Icarus 168(2), 457-466.

Buczkowski, D. L., O. S. Barnouin-Jha, and L. M. Prockter (2008), 433 Eros lineaments: Global mapping and analysis, Icarus 193(1), 39-52.

Buczkowski, D. et al. (2011), Structural features on 4Vesta: Observations and analysis, Abstract U21B-05, presented at 2011 Fall Meeting, AGU, San Francisco, Calif., 5-9 Dec.

Buczkowski, D.L. et al. (2012), Large-scale troughs on Vesta: A signature of planetary tectonics, Geophysical Research Letters, 39, L18205, doi: 10.1029/2012GL052959.

Burbine, T. H., P. C. Buchanan, R. P. Binzel, S. J. Bus, T. Hiroi, J. L. Hinrichs, A. Meibom, and T. J. McCoy (2001), Vesta, Vestoids, and the howardite, eucrite, diogenite group: Relationships and the origin of spectral differences, Meteoritics \& Planetary Science, 36(6), 761-781.

Cohn, S. N., and T. J. Ahrens (1981), Dynamic tensile strength of lunar rock types, Journal of Geophysical Research, 86, 1794-1802.

Crawford, D.A. (1999), Adaptive Mesh Refinement in CTH. Paper presented at 15th US Army Symposium on Solid Mechanics, Myrtle Beach, SC, April 12-14.

Crawford, D.A. et al. (2006), Adaptive Mesh Refinement in the CTH Shock Physics Hydrocode, The Russian Journal of Physical Chemistry B, 25:9, 85-90.

Davis, D. R., C. R. Chapman, S. J. Weidenschilling, and R. Greenberg (1985), Collisional history of asteroids: Evidence from Vesta and the Hirayama families, Icarus, 62(1), 30-53.

Davis, D.R., and E.V. Ryan (1990) On collisional disruption: Experimental results and scaling laws. Icarus, 125, 50-60.

Davis, D.R., E.V Ryan, P. Farinella (1994) Asteroid collisional evolution: results from current scaling algorithms. Planet. Space Sci., 42(8), 599-610.

de Joussineau, G., J.-P. Petit, and B.D.M. Gauthier (2003), Photoelastic and numerical investigation of stress distributions around fault models under biaxial compressive loading conditions. Tectonophysics 363(1-2), 19-43.

Ermakov, A.I., M.T. Zuber, D.E. Smith, C.A. Raymod, D. Balmino, R.R. Fu, and B.A. Ivanov (2014) Constraints on Vesta's Interior Structure Using Gravity and Shape Models from the Dawn Mission, Icarus, in press. doi: 10.1016/j.icarus.2014.05.015. 
Dreibus, G., J. Bruckner, and H. Wanke (1997), On the core mass of the asteroid Vesta., Meteoritics \& Planetary Science, 32(4), A36-A36.

Feierberg, M. A., and M. J. Drake (1980), The Meteorite-Asteroid Connection - the InfraredSpectra of Eucrites, Shergottites, and Vesta, Science, 209(4458), 805-807.

Feierberg, M. A., H. P. Larson, U. Fink, and H. A. Smith (1980), Spectroscopic Evidence for 2 Achondrite Parent Bodies - Asteroids 349-Dembowska and 4-Vesta, Geochimica et Cosmochimica Acta, 44(3), 513-524.

Fujiwara, A. (1986) Results Obtained by Laboratory Simulations of Catastrophic Impact, Mem. S.A.It., 57(1), pp. 47-64.

Fujiwara, A., and N. Asada (1983), Impact Fracture Patterns on Phobos Ellipsoids, Icarus, 56(3), 590-602.

Fujiwara, A., P. Cerroni, D. David, E. Ryan, and M di Martino (1989), Experiments and scaling laws for catastrophic collisions, in: Asteroids II: Proceedings of the Conference, Tucson AZ, Mar. 8-11. University of Arizona Press: Tucson, AZ, pp. 240-265.

Fujiwara, A., and A. Tsukamoto (1980), Experimental study on the velocity of fragments in collisional breakup, Icarus, 44(1), 142-153.

Gaffey, M. J. (1997), Surface lithologic heterogeneity of asteroid 4 Vesta, Icarus, 127(1), 130157.

Gault, D.E (1973) Displaces mass, depth, diameter, and effects of oblique trajectories for impact craters formed in dense crystalline rocks. The Moon, 6, 32-44.

Gault, D. E., and J. A. Wedekind (1969), The Destruction of Tektites by Micrometeoroid Impact, J. Geophys. Res., 74.

Gault, D.E., and J.A. Wedekind (1978), Experimental studies of oblique impact, paper presented at 9th Lunar and Planetary Science Conference, 1978.

Hartmann, W.K. (1980) Continued low-velocity impact experiments at Ames Vertical Gun Facility: miscellaneous results. Lunar and Planetary Science Conference 19: 451-52.

Holsapple, K. A. (1993), The Scaling of Impact Processes in Planetary Sciences, Annual Review of Earth and Planetary Sciences, 21, 333-373.

Holsapple, K., I Giblin, K. Housen, A. Nakamura, and E. Ryan (2002), Asteroid impacts: Laboratory experiments and scaling laws. In: Asteroids III (eds. W.F. Bottke, A. Cellina, P Paolicchi, and R.P. Binzel). The University of Arizona Press: Tuscon, AZ., pp. 443-464.

Housen, K. R., R. M. Schmidt, and K. A. Holsapple (1983), Crater ejecta scaling laws Fundamental forms based on dimensional analysis, Journal of Geophysical Research, 88, 2485-2499.

Hughes, H.G., F.N. App, and T.R. McGetchin (1977), Global Seismic Effects of Basin-forming Impacts, Phys. Earth Planet. Int. 15, 251-263.

Ivanov, B.A. and H.J. Melosh (2013), Two-dimensional numerical modeling of the Rheasilvia impact formation. Journal of Geophysical Research Planets, 118, 1545-1557, doi: 10.1002/jgre.20108.

Jaumann, R. et al. (2012) Vesta's shape and morphology, Science, 336, 687-690, doi:10.1126/science.1219122.

Johnson, G.R., and W.H. Cook (1985), Fracture characteristics of three metals subjected to various strains, strain rates, temperatures and pressures. Engr. Fracture Mech. 21, 31-48.

Konopliv, A.S. et al. (2013) The Vesta gravity field, spin pole and rotation period, landmark position, and ephemeris from the Dawn tracking and optical data, Icarus, in press. doi: 10.1016/j.icarus.2013.09.005. 
Le Corre, L., V Reddy, A. Nathues, D.A Williams, W.B. Garry, R.A. Yingst, R. Jaumann, T Roatsch, F. Preusker, C.M. Pieters, C.T. Russell, and C.A. Raymond (2012), Geologic Mapping of the AV-6 (Gegania) Quadrangle of Asteroid 4 Vesta, LPS XLIII, Abstract \#1629.

Martelli, G., E.V. Ryan, A.M. Nakamura, and I Giblin (1994), Catastrophic Disruption Experiments: Recent results, Planet. Space Sci., 42, 1013-1026.

Marsh, S. P. (1980), LASL shock Hugoniot data. 394 pp., Berkeley and Los Angeles.

McCord, T. B., J. B. Adams, and T. V. Johnson (1970), Asteroid Vesta: Spectral Reflectivity and Compositional Implications, Science, 168(3938), 1445-1447.

McFadden, L. A., T. B. McCord, and C. Pieters (1977), Vesta: The first pyroxene band from new spectroscopic measurements, Icarus, 31(4), 439-446.

McGlaun, J.M., S.L. Thompson, and M.G. Elrick (1990), CTH: A three-dimensional shock wave physics code. Int. J. Imp. Engr. 10(1-4), 351-360.

Melosh, H. J., E. V. Ryan, and E. Asphaug (1992), Dynamic Fragmentation in Impacts: Hydrocode Simulation of Laboratory Impacts, J. Geophys. Res., 97(E9), 14735-14759.

Melosh, H. J., and E. V. Ryan (1997), Asteroids: Shattered but Not Dispersed, Icarus, 129(2), 562-564.

Meschede, M. A., C. L. Myhrvold, and J. Tromp (2011), Antipodal focusing of seismic waves due to large meteorite impacts on Earth, Geophysical Journal International, 187(1), 529537.

Michalak, G. (2000), Determination of asteroid masses I. (1) Ceres, (2) Pallas and (4) Vesta, Astron Astrophys, 360(1), 363-374.

Misra, S., N. Mandal, R. Dhar, and C. Chakraborty (2009), Mechanisms of deformation localization at the tips of shear fractures: Findings from analogue experiments and field evidence. J. of Geophys. Res. (Solid Earth) 114, 04204.

Moore, J.M., P.M. Schenk, L.S. Bruesch, E. Asphaug, and W.B. McKinnon (2004), Large impact features on middle-sized icy satellites, Icarus 171(2), 421-443.

Nakamura, A., and A. Fujiwara (1991), Velocity distribution of fragments formed in a simulated collisional disruption, Icarus, 92(1), 132-146.

Nakamura, A.M., A. Fujiwara, T. Kadono, and S. Shirono (1994) Fragmentation of Intact and Pre-Impacted Basalt Targets. In: Seventy-Five Years of Hirayama Asteroid Families SP Conference Series, (eds. Y. Kozai, R.P Binzel, and T. Hirayama), vol 63.

Nasraoui, M., P. Forquin, L. Siad, and A. Rusinek (2012) Influence of strain rate, temperature and adiabatic heating on the mechanical behaviour of poly-methyl-methacrylate:

Experimental and modelling analysis, Materials and Design 37, 500-509.

Nolan, M.C., E. Asphaug, R. Greenberg, and H.J Melosh (2001). Impacts on Asteroids: Fragmentation, Regolith Transport, and Disruption. Icarus, 153, 1-15.

Righter, K., and M. J. Drake (1997), A magma ocean on Vesta: Core formation and petrogenesis of eucrites and diogenites, Meteoritics \& Planetary Science, 32(6), 929-944.

Rinehart, J.S. (1960), Stress Transients in Solids. Hyperdynamics: Santa Fe, NM.

Rittel, D., and A. Brill (2008), Dynamic flow and failure of confined polymethylmethacrylate. $J$. of the Mech. and Phys. of Solids 56, 1401-1416.

Rosakis, A.J. (2002), Intersonic shear cracks and fault ruptures. Advances in Phys. 51(4), 11891257.

Rosakis, A.J., O. Samudrala, and D. Coker (1999), Cracks Faster than the Shear Wave Speed. Science 284(5418), 1337-1340. 
Rosakis, A.J., H. Kanamori, K. Xia, and J.R. Rice (2004), Laboratory earthquakes in bimaterial systems: on the directionality of the Parkfield earthquakes. EOS Trans. AGU 85(47), Fall Meet. Suppl., Abstract S54B-07.

Rosakis, A.J., H.S. Bhat, C.G. Sammis, and R.L. Biegel (2008), The Effect of Asymmetric Damage on Dynamic Shear Rupture Propagation With Mismatch in Bulk Elasticity. EOS Trans. AGU 89(53), Fall Meet. Suppl., Abstract S51D-1775.

Russell, C.T., et al. (2007), Dawn Mission to Vesta and Ceres, Earth, Moon, and Planets, 101(1), 65-91.

Russell, C.T., et al. (2012) Dawn at Vesta: Testing the Protoplanetary Paradigm, Science 336, 684-686. Doi:10.1126/science.1219381

Ruzicka, A., G. A. Snyder, and L. A. Taylor (1997), Vesta as the howardite, eucrite and diogenite parent body: Implications for the size of a core and for large-scale differentiation, Meteoritics \& Planetary Science 32(6), 825-840.

Ryan, E. V., and H. J. Melosh (1998), Impact Fragmentation: From the Laboratory to Asteroids, Icarus 133(1), 1-24.

Schenk, P., S. Marchi, D.P. O’Brien, D. Buczkpwski, R. Jaumann, A. Yingst, T. McCord, R. Gaskell, T. Roatsch, H.E. Keller, C.A. Raymond, and C.T Russell (2012a), Mega-impacts into Planetary Bodies: Global Effects of the Giant Rheasilvia Impact Basin on Vesta, LPS XLIII, Abstract \#2757.

Schenk, P., et al. (2012b) The Geologically Recent Giant Impact Basins at Vesta's South Pole, Science, 336(6082), 694-697, doi: 10.1126/science.1223272.

Schultz, P.H., and D.E. Gault (1975), Seismic effects from major basin formations on the moon and mercury, Earth, Moon, and Planets 12(2), 159-177.

Schultz, P.H., and D.A. Crawford (2011) Origin of nearside structural and geochemical anomalies on the Moon, in: Recent Advances and Current Research Issues in Lunar Stratigraphy, GSA Special Paper 477 (eds. W.A. Ambrose, and D.A. Williams). The Geological Society of America: Boulder, CO.

Scully, J.E.C., C.T. Russell, A. Yin, D.A. Williams, D.T. Blewett, D.L. Buczkowski, E. Ammannito, T. Roatsch, F. Preusker, L. Le Corre, R.A. Yingst, W.B. Garry, R Jaumann, C.M. Pieters, and C. A Raymond (2012), Geologic Mapping of the AV-4 Domitia Quadrangle of Asteroid 4 Vesta, LPS XLIII, Abstract \#2368.

Session, S., and M. Wysession (2003), An introduction to seismology, earthquakes, and earth structure, Blackwell Publishing Ltd.: Malden, MA.

Stickle, A.M., and P.H. Schultz (2010), Comparing Experimental and Numerical Results for Subsurface Failure Following Oblique Impacts into Planar Targets, LPS $X L I$, Abstract \#2598.

Stickle, A.M., and P.H. Schultz (2011), Exploring the role of shear in oblique impacts: A comparison of experimental and numerical results for planar targets. Int. J. Imp. Engr. 38(6), 527-534.

Stickle, A.M. and P.H. Schultz (2012), Subsurface damage from oblique impacts into lowimpedance layers, Journal of Geophysical Research, 117, E07006, doi: 10.1029/2011JE004043.

Stickle, A.M. and P.H. Schultz (2013) Investigating Pressure Magnitudes at Depth for Oblique Impacts into Layered Targets: Applications to Terrestrial Impacts in Sedimentary Targets, Meteoritics \& Planetary Science, 1-13, doi: 10.1111/maps.12152. 
Stickle, A.M. and P.H. Schultz (2014) Discrete Shear Failure Planes Resulting from Oblique Hypervelocity Impacts, Journal of Geophysical Research - Planets, 119, doi: 10.1002/2013JE004597.

Takagi, Y., H. Mizutani, and S.-I. Kawakami (1984), Impact fragmentation experiments of basalts and pyrophyllites, Icarus, 59(3), 462-477.

Thomas, P. C., R. P. Binzel, M. J. Gaffey, A. D. Storrs, E. N. Wells, and B. H. Zellner (1997), Impact excavation on asteroid 4 Vesta: Hubble Space Telescope results, Science, 277(5331), 1492-1495.

Thompson, S.L. (1990), ANEOS analytic equations of state for shock physics codes input manual. Rep. SAND-89-2951, Sandia National Labs, Albuquerque, NM.

Thompson, S.L., and H.S. Lauson (1984), Improvements in the Chart-D radiation hydrodynamic CODE. III. Revised analytic equations of state. Rep. SC-RR-710714, Sandia National Labs, Albuquerque, NM.

Williams, J.G. (1989), Asteroid family identifications and proper elements. In: Asteroids II (eds. R.P Binzel, T. Gehrels, and M.S. Mathews), University of Arizona Press: Tucson, AZ. pp. 1034-1072,

Zappalà, V., Ph. Bandjoya, A. Cellino, P Farinella, and C. Froeschlé (1995) Asteroid families: Search of 12,487-asteroid sampleusing two different clustering techniques. Icarus, 116, 291314. 